\title{
THE r-, s-, AND p-PROCESSES IN NUCLEOSYNTHESIS
}

\author{
Bradley S. Meyer \\ Department of Physics and Astronomy, Clemson University, Clemson, South \\ Carolina 29634-1911
}

KEY WORDS: heavy elements, supernovae, AGB stars, abundances

\section{INTRODUCTION}

Burbidge et al (1957) and Cameron (1957) laid out the framework for our understanding of the formation of the heavy nuclei (those nuclei with mass number $A \gtrsim 70$ ). From systematics in the solar system abundance distribution, Burbidge et al determined that the heavy nuclei were formed in three distinct nucleosynthetic processes, which they termed the $\mathrm{r}-, \mathrm{s}-$, and $\mathrm{p}$-processes. That we still use these terms today is a credit to the soundness of this work done 37 years ago.

We may understand how Burbidge et al and Cameron arrived at their conclusions from Figure 1. One population of nuclei, the s-nuclei, shows an abundance distribution with peaks near mass numbers 87,138 , and 208 . These nuclei are made in a slow neutron-capture process, the s-process. A rapid neutron-capture process, the r-process, is responsible for the r-nuclei, whose abundance distribution shows peaks at mass numbers 80,130, and 195. The p-process is responsible for production of the rarer, more proton-rich heavy isotopes (the p-nuclei) that cannot be made by neutron capture.

The first quantitive evaluations of the ideas of Burbidge et al and Cameron came to light in the early 1960s with work on the s-process (Clayton et al 1961, Seeger et al 1965) and the r-process (Seeger et al 1965). These calculations further elucidated the mechanisms for heavy-element formation and showed the plausibility of the framework developed in the 1950s. Subsequent work has focused on determining the astrophysical sites where the r-, s-, and pprocesses occurred with the help of improved nuclear details, stellar models, and abundances. A goal of this paper is to review the recent progress astrophysicists, 


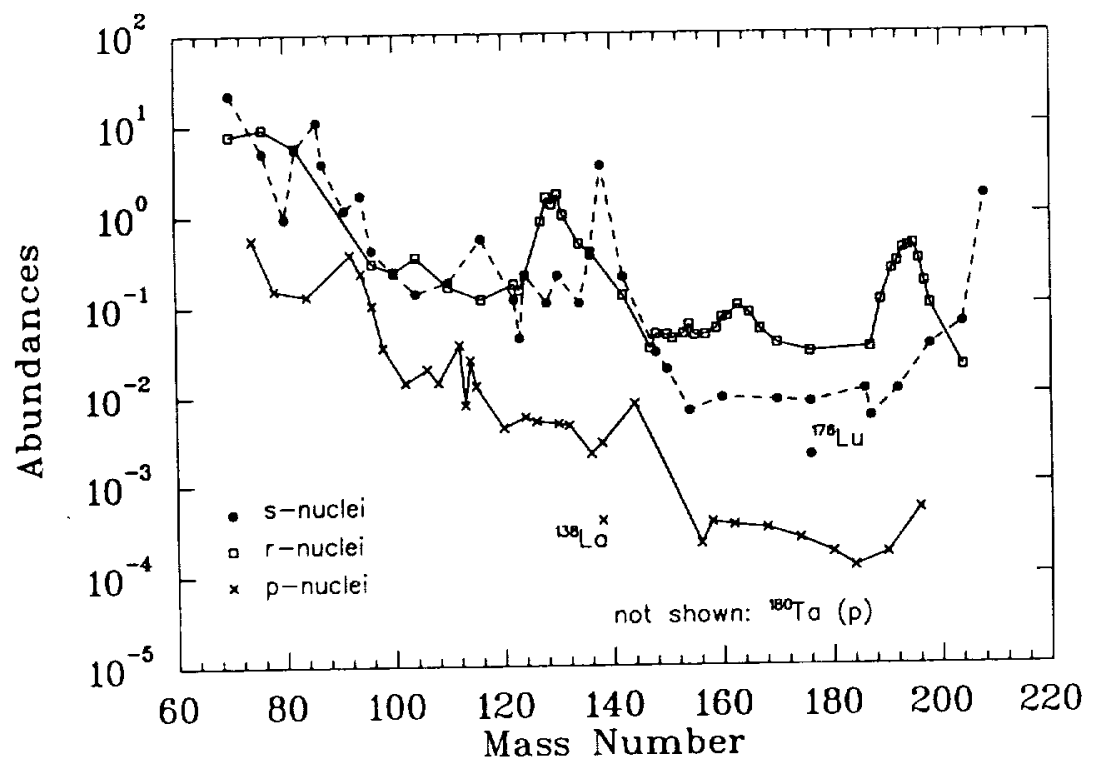

Figure 1 The solar system abundances of r-nuclei, s-nuclei, and p-nuclei, relative to $\mathrm{Si}=10^{6}$. Only isotopes for which $90 \%$ or more of the inferred production comes from a single process are shown. The data are from Anders \& Grevesse (1989) and Käppeler et al (1989).

astronomers, and physicists have made in these directions and to point out the problems that remain in our understanding of the formation of the heavy nuclei. Another, perhaps deeper, goal is to to seek some understanding of why there are three major processes available to nature for synthesis of heavy elements.

It is impossible for a single paper to cover all relevant aspects of the r-, s-, and p-processes; therefore, where possible, references to other reviews are given. Readers should turn to these reviews for more details. Nevertheless, it is hoped that the present paper gives some flavor for the rich field of heavy-element synthesis.

\section{GENERAL CONSIDERATIONS}

The Master said, "Ssu, I believe you look upon me as one whose aim is simply to learn and retain in mind as many things as possible." He replied, "That is what I thought. Is it not so?" The Master said, "No; I have one thread on which I string them all."

The Analects of Confucius 
The r-, s-, and p-processes are distinct nucleosynthetic mechanisms. They occur in different environments and under quite different conditions. Nevertheless, it is useful to seek some unifying concept by which we may understand these disparate processes. We will see that entropy is the concept we need. Careful consideration of entropy in the various nucleosynthetic processes will clarify our discussion and give us insight into how these processes occur and why they occur where they do. With this insight we will see how the r-, s-, and p-processes are each unique answers to the same question: "How does nature produce heavy elements?"

\subsection{Entropy and Equilibrium}

Let us begin our discussion by considering a given thermally isolated system at constant volume. The system has total energy $E_{0}$. The entropy of this system is

$$
S=k \ln \Gamma,
$$

where $k$ is Boltzmann's constant and $\Gamma$ is the number of energetically allowed macroscopic states available to the system. By macroscopic state we mean a particular distribution of the constituents of the system among their singleparticle quantum-mechanical states. In essence, a macroscopic state of the system is one particular way the constituents of the system can share the total energy $E_{0}$. Suppose the system does not have all macroscopic states of energy $E_{0}$ available to it. In this case, the entropy is less than its maximum. The system will evolve by the Second Law of Thermodynamics and add more macroscopic states to its repertoire. In this evolution, the entropy will thus increase. The system will continue to evolve until all macroscopic states of energy $E_{0}$ are available to the system. Once the system reaches this point, it is at maximum entropy and experiences no further evolution. The system has attained equilibrium. We thus see that equilibrium, or maximum entropy, is the evolutionary endpoint of any thermally isolated system.

We can now use these considerations to ask what happens to the nucleons and nuclei in some nucleosynthetic environment. If the system of nucleons and nuclei is thermally isolated and out of equilibrium, it will evolve towards equilibrium. Given enough time, the system will reach equilibrium and attain maximum entropy. At this point, it is in nuclear statistical equilibrium (NSE), and it is a simple matter to compute the abundance of any nuclide (Burbidge et al 1957). We find (e.g. Meyer 1993) that, for a nuclear species of atomic number $Z$ and mass number $A$, the abundance per baryon $Y(Z, A)$ is

$$
\begin{aligned}
Y(Z, A)= & G(Z, A)\left[\zeta(3)^{A-1} \pi^{(1-A) / 2} 2^{(3 A-5) / 2}\right] A^{3 / 2}\left(\frac{k T}{m_{\mathrm{N}} c^{2}}\right)^{3(A-1) / 2} \\
& \times \phi^{1-A} Y_{\mathrm{p}}^{Z} Y_{\mathrm{n}}^{A-Z} e^{\left[\frac{B(Z, A)}{k T}\right]},
\end{aligned}
$$


where $G(Z, A)$ is the nuclear partition function, $\zeta(3)$ is the Riemann zeta function of argument $3, T$ is the temperature, $m_{N}$ is the mass of a single baryon, $\phi$ is the photon-to-baryon ratio, $Y_{\mathrm{p}}$ is the abundance per baryon of protons, $Y_{\mathrm{n}}$ is the abundance per baryon of neutrons, and $B(Z, A)$ is the binding energy of nucleus $(Z, A)$. We note that $\phi$ is given by

$$
\phi=\frac{2}{\pi^{2}} \frac{1}{(\hbar c)^{3}} \frac{\zeta(3)(k T)^{3}}{\rho \mathcal{N}_{A}} .
$$

where $\mathcal{N}_{A}$ is Avagadro's number and $\rho$ is the baryon mass density. The binding energy of nucleus $(Z, A)$ is

$$
B(Z, A)=\left[Z m_{\mathrm{p}}+N m_{\mathrm{n}}-m(Z, A)\right] c^{2},
$$

where $N=A-Z$ and $m(Z, A), m_{\mathrm{p}}$, and $m_{\mathrm{n}}$ are the masses of nucleus $(Z, A)$, the proton, and the neutron, respectively.

From Equation (2), we see that the NSE abundance of nuclei is nonzero. This might at first be surprising. If we combine free neutrons and protons into nuclei, we decrease the number of free particles of the system. This would yield fewer ways of sharing the total energy of the system and thus decrease the number of macroscopic states available to the system. The nuclear reactions also release binding energy, however, which increases the number of photons in the system, the energy available to leptons, and the excitation energy in the nuclei. These effects increase the number of ways the system can share the total energy of the system and, hence, can increase the number of macroscopic stites available to the system. This increase can more than compensate for the decrease in the number of states due to the loss of free particles and can lead to an increase in the entropy. Once the system has evolved to the point that it experiences no net increase in the number of macroscopic states by changing the abundance of any particular nucleus, the system has reached NSE.

Which nuclei dominate the abundance distribution in NSE? This depends on the photon-to-baryon ratio or, equivalently, the entropy per baryon since this latter quantity scales monotonically with the photon-to-baryon ratio (e.g. Meyer \& Walsh 1993). From Equation (2) we see that the abundance of some heavy nucleus $(Z, A)$ depends on $\phi^{1-A}$. At some given temperature then, the larger $\phi$ is, the smaller will be the abundance of the heavy nucleus $(Z, A)$. Fewer heavy nuclei means more light nuclei and free nucleons. The strong dependence of the NSE abundances on $\phi$ will be crucial for the r-process. This dependence on $\phi$ is apparent in Figure 2. If we fix the temperature, the larger $\phi$ is, the more likely nucleons are free or contained in light nuclei.

\subsection{Equilibrium Nucleosynthesis}

We have seen that the goal of any nucleosynthetic process is equilibrium. How does this goal vary with temperature? The answer is important because nature 


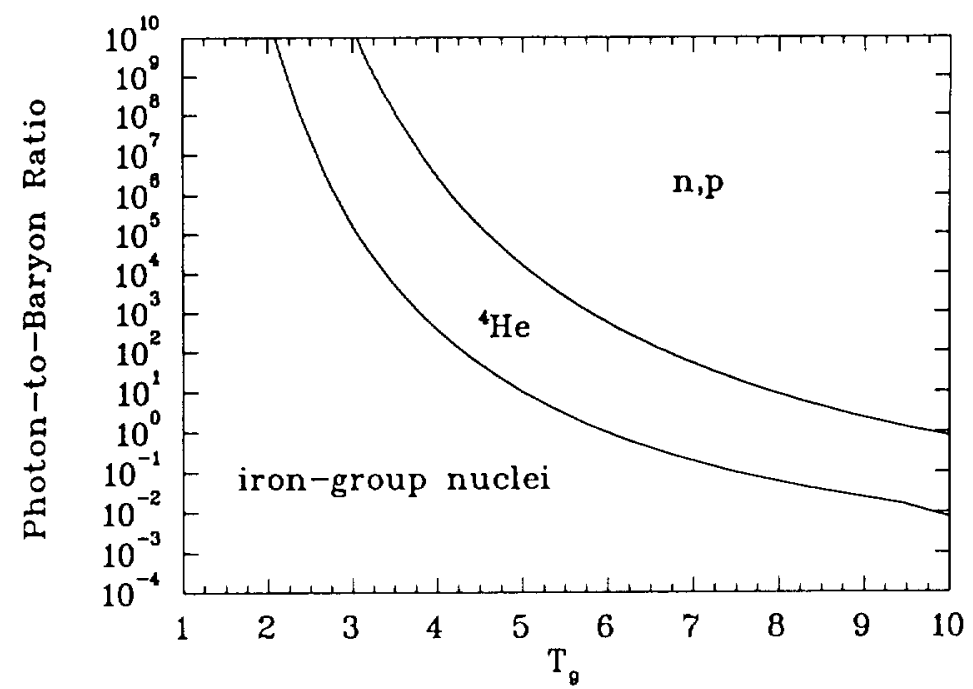

Figure 2 The dominant species in NSE for a gas with electron-to-baryon ratio $Y_{\mathrm{e}}=0.5$. The different regions of the plot show at what temperatures and photon-to-baryon ratios $\phi$ the various species dominate the gas. At high temperatures and high photon-to-baryon ratios, free neutrons and protons (labeled $n, p$ ) dominate. At lower temperatures and photon-to-baryon ratios, the nucleons are mostly locked up in ${ }^{4} \mathrm{He}$ nuclei. At still lower temperatures and photon-to-baryon ratios, the gas will predominantly be a distribution of iron-group nuclei.

synthesizes nuclei at high temperature and ejects them into the medium between the stars at low temperature. If the nuclei stayed in equilibrium throughout this processing, we could use Equation (2) to determine the abundance of the various species. That the nuclei emerging from some nucleosynthetic process are not in general in an equilibrium distribution gives us important clues about how they were in fact made. We will turn to this point after discussing equilibrium nucleosynthesis in some detail.

Consider a system in NSE at some temperature. If the system expands adiabatically, the temperature will decrease. By Le Chatelier's Principle we know the system will respond to this stress by tending to counter it. How does the system do this? The answer is that nuclear reactions occur and release binding energy. This energy heats up the system and tends to counter the temperature decrease from the expansion. Via these nuclear reactions, the system finds a new equilibrium if the reactions occur faster than the expansion.

As we have noted in Section 2.1, if nuclear reactions assemble free particles and light nuclei into heavier nuclei, there is a loss of macroscopic states and a decrease in the entropy in baryons (free nucleons and nuclei). The binding energy released in the reactions goes into photons and leptons. This increases 
the numbers of macroscopic states and, hence, the entropy in the photons and leptons. We see that in building up the abundances of heavy nuclei, we transfer entropy from the baryons to the photons and leptons. This is apparent in Figure 3. Suppose a system at an entropy per baryon of $10 k$ begins in NSE at $T_{9}=10\left(T_{9} \equiv T / 10^{9} \mathrm{~K}\right)$ and expands adiabatically. The system cools. Because the system maintains equilibrium, entropy leaves the baryons as the baryons assemble themselves into heavier nuclei via nuclear reactions. This entropy goes into the photons and leptons (electron-positron pairs).

The entropy in baryons continues to fall until $T_{9} \approx 4$. At this point, the system has released essentially all its nuclear binding energy. There is little further transfer of entropy from the baryons to the other constituents of the system. We also note that beginning around $T_{9}=4$ the electron-positron pairs annihilate into two photons. This transfers entropy from the $e^{+}-e^{-}$pairs into the photons adiabatically. At late times, after the $e^{+}-e^{-}$pairs have annihilated, the system is left with a residual abundance of electrons to ensure charge neutrality. After the pairs have disappeared, the system is a mix of relativistic photons and nonrelativistic nuclei and electrons. Now for adiabatic expansion, $\rho \propto T^{3}$ for relativistic particles and $\rho \propto T^{3 / 2}$ for nonrelativistic particles. The system at late times, because it is a mix of these two types of particles, actually expands such that $\rho \propto T^{b}$ where $b$ is between $3 / 2$ and 3 . This means that entropy now is transferred from the photons to the translational

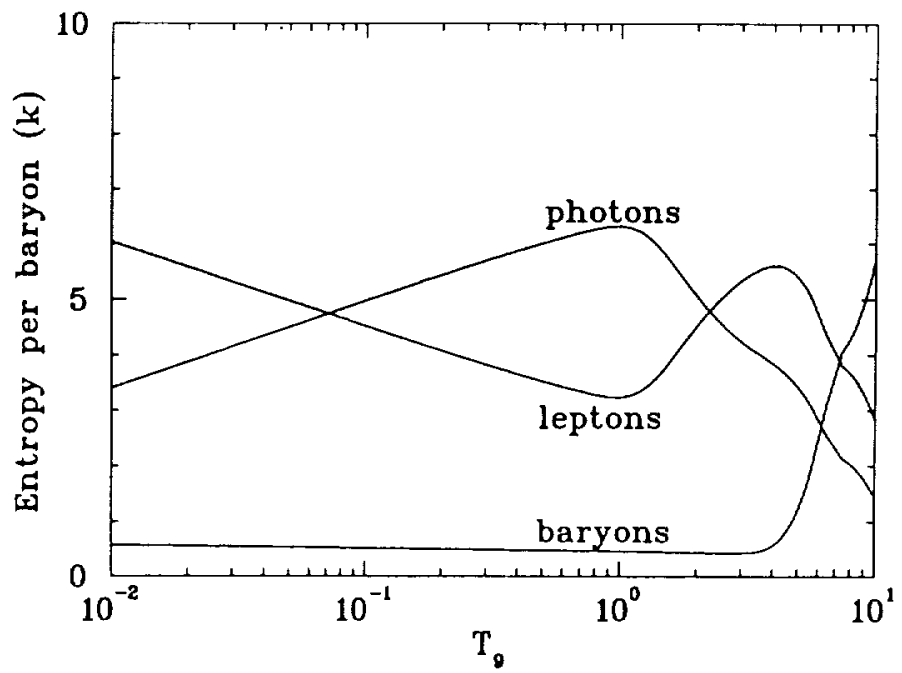

Figure 3 The entropy per baryon in the consitituents of a system expanding adiabatically with total entropy per baryon $10 k$ and electron-to-baryon ratio $Y_{c}$. Note that the sum of the entropies of the constituents is always $10 k$. 
entropy of the nonrelativistic particles. This is why the entropy in photons declines after $T_{9}=1$ and that in leptons and baryons rises.

What nuclei are made in such an expansion? Again we note that nuclear reactions tend to occur only if there is a release of binding energy to compensate for the loss of macroscopic states due to the decrease in the number of free particles. As our system expands and cools, the nuclei present in equilibrium will increase in mass. They will continue to increase in mass until they have reached the nuclide with the largest binding energy per nucleon. Once this nuclide dominates the abundance of nuclei, there is no further evolution in the abundances. This is because if the system attempts to arrange the nucleons into a more massive nucleus with a lower binding energy per nucleon, there will be a net decrease in the total binding energy of the system. Reactions giving this rearrangement of nucleons lead to a decrease in the number of free particles and in the energy available to the photons and leptons. There will thus be fewer ways to share the energy of the system and a decrease in the entropy. An expanding and cooling system can maintain NSE by driving the system to a composition dominated by the nuclear species with the largest binding energy per nucleon.

Figure 4 shows the mass fraction $X(Z, A)=A Y(Z, A)$ in NSE at temperatures $T_{9}=1-10$ for an entropy per baryon of $10 k$ and a net electron-to-baryon ratio $Y_{\mathrm{e}}=0.5$. If we imagine the system begins at $T_{9}=10$, alpha particles

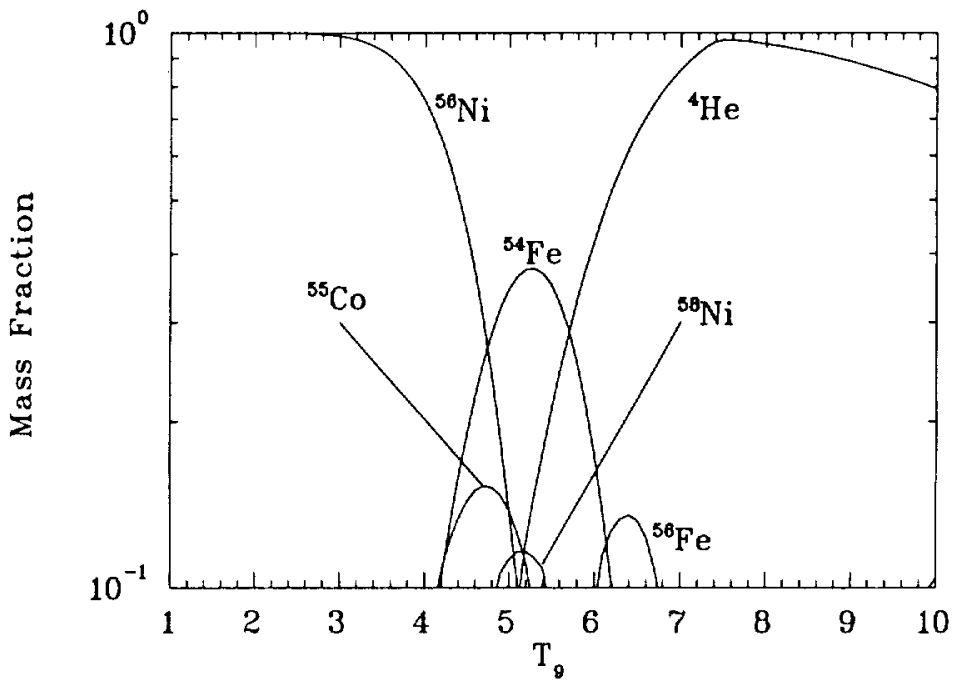

Figure 4 The mass fraction of species in NSE as a function of temperature for a system with entropy per baryon $10 k$ and $Y_{c}=0.5$. 
dominate the mass of the system. As the system expands and cools adiabatically, the abundances of heavier nuclei increase. At late times, however, all nucleons are locked up in ${ }^{56} \mathrm{Ni}$ nuclei. This is because ${ }^{56} \mathrm{Ni}$ has one proton for every two nucleons $\left(Y_{\mathrm{e}}=0.5\right)$, and it is the nucleus at $Y_{\mathrm{e}}=0.5$ with the strongest binding energy per nucleon.

Figure 5 shows what happens if the entropy per baryon is $100 k$ instead of $10 k$. Because of the larger entropy, the system has more free particles and light nuclei at a given temperature. This is why free neutrons and protons dominate the abundances around $T_{9}=10$ in Figure 5 while alpha particles dominate the abundances in Figure 4. We also see that alpha particle abundances build up only after $T_{9}$ drops below 10 for entropy per baryon of $100 k$. As in the lower entropy case, however, the nucleons eventually end up all in ${ }^{56} \mathrm{Ni}$.

What happens now if $Y_{\mathrm{e}}$ is different from 0.5 ? Figure 6 shows what happens if the entropy per baryon is $10 k$ and $Y_{\mathrm{e}}=0.4$. Here there are six neutrons for every four protons. We see that the system at late times locks up all its nucleons into ${ }^{70} \mathrm{Ni}$. For $Y_{\mathrm{e}}=0.4$, this is the nucleus with the largest binding energy per nucleon.

From Figure 6 we see that the final nucleus on which the system converges at low temperature is dependent on $Y_{\mathrm{e}}$ because the final nuclei present must accommodate all of the neutrons and protons initially in the system. This final nucleus, however, is the nucleus with the largest binding energy for that $Y_{\mathrm{e}}$.

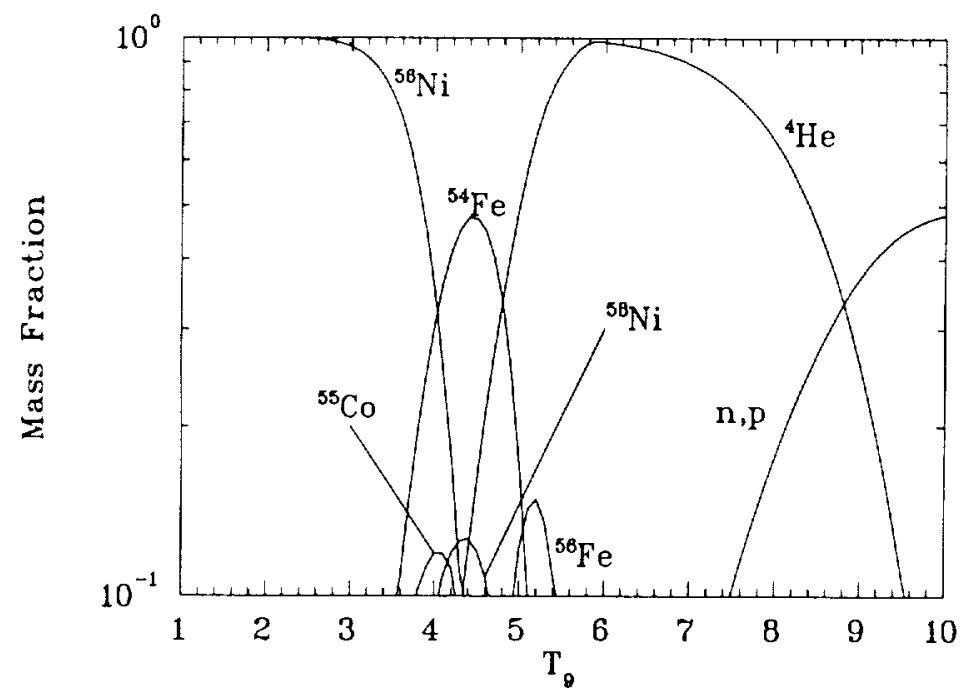

Higure 5 The mass fraction of species in NSE as a function of temperature for a system with entropy per baryon $100 \mathrm{k}$ and $Y_{\mathrm{e}}=0.5$. 


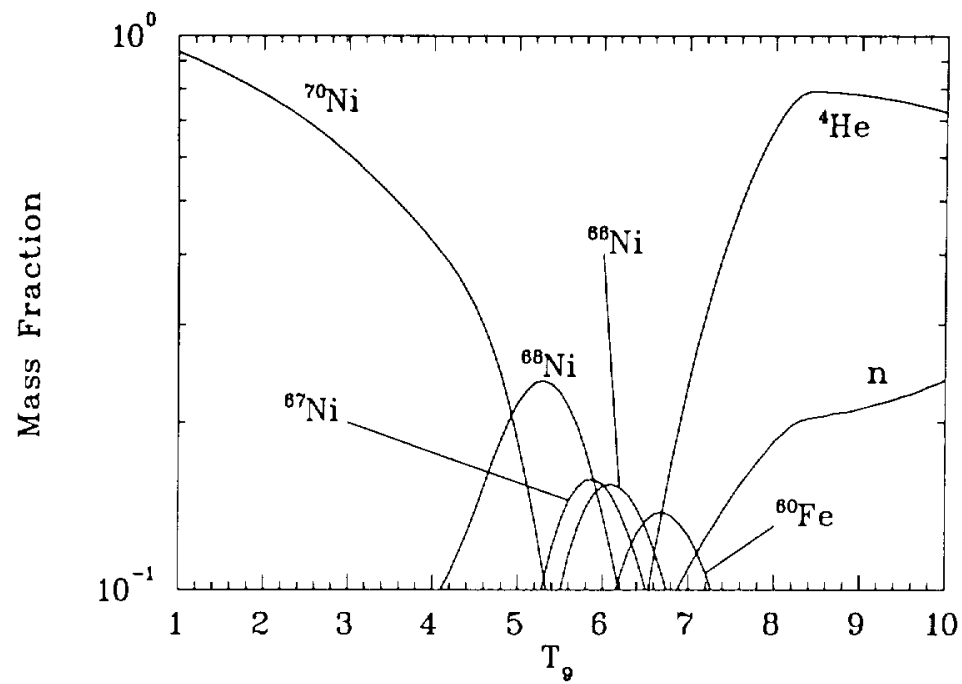

Figure 6 The mass fraction of species in NSE as a function of temperature for a system with entropy per baryon $10 k$ and $Y_{\mathrm{e}}=0.4$.

Figure 7 gives an idea of what that nucleus is for $Y_{\mathrm{e}}$ ranging from 0.35 to 0.6 . In all cases, the nuclei are iron-group nuclei $(Z=26-34)$.

\subsection{How to Make Heavy Nuclei}

We are now in a position to ask the question "How does nature make heavy nuclei?" We have seen in the previous sections that if we eject nuclei into cold interstellar space, and if these nuclei are always in NSE, then these nuclei must be those with the largest binding energy per nucleon for whatever $Y_{\mathrm{e}}$ is appropriate for the environment in which the nuclei find themselves. From Figure 7, however, we see that for a large range of $Y_{\mathrm{e}}$, these nuclei are simply iron-group nuclei. If NSE always pertains, stars and supernovae can only eject iron-group nuclei. Our observations of uranium on Earth tell us that this is not what happens.

The escape from this dilemma is the fact that nucleosynthetic systems cannot always be in NSE. There are only two ways this can happen. The first possibility is that the system never has time to come into NSE before the star ejects the nucleons into the interstellar medium. In this case the nucleons assemble themselves part of the way up to iron. This is the "falling short of equilibrium" scenario. The second possibility is that the system begins in NSE at high temperature. As the system expands and cools, the equilibrium changes. As the temperature drops, some nuclear reactions slow down. Eventually these 

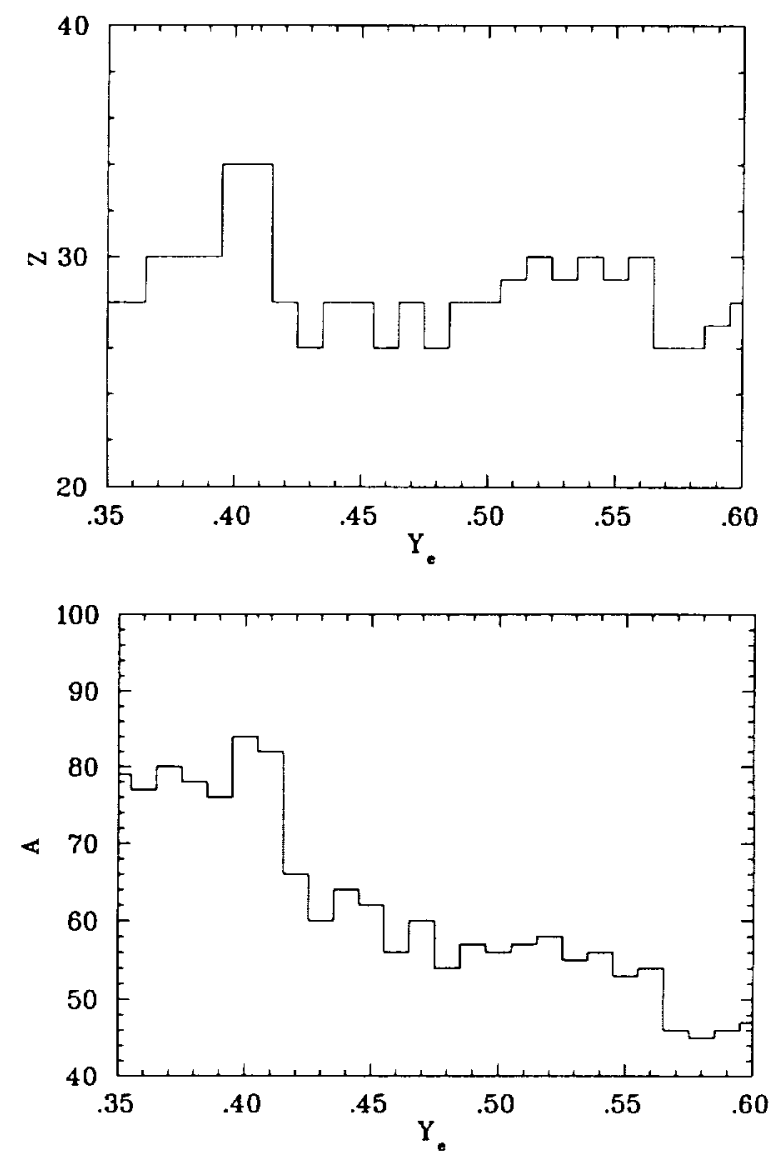

Figure 7 The proton number $Z(t o p)$ and the mass number $A$ (bottom) of the species with the largest binding energy per nucleon in each bin in $Y_{e}$. Where experimental data are available, they are from Wapstra et al (1988). Where experimental data are unavailable, nuclear masses are from Möller \& Nix (1988).

reactions become too slow to allow the system to maintain equilibrium. This is the "freezeout from equilibrium" scenario. These are the only two ways a nucleosynthetic system can be out of equilibrium, and they are the only two options nature has to assemble heavy nuclei.

How in fact does nature make heavy elements in these two scenarios? In the freezeout from equilibrium, a nucleosynthetic system falls out of equilibrium. At this point the composition reflects the last NSE abundance distribution the system attained before it fell out of equilibrium. This composition is a mix of free nucleons, light nuclei, and iron-group nuclei. While some of the reactions 
necessary to maintain equilibrium become too slow, others such as capture of free nucleons and light nuclei on iron-group nuclei can continue. The irongroup nuclei can serve as "seeds" for the capture of the remaining free nucleons and light nuclei. These captures can produce heavy nuclei. Because the system assembles its own seed nuclei in such a scenario, the system can make heavy elements without any pre-existing seed nuclei. A process that does not require pre-existing seed nuclei to make new nuclei is called primary.

In the other scenario- the falling short of equilibrium scenario-the material never achieves NSE because the timescale to reach equilibrium is always too long compared to the dynamical timescale of the system. The system assembles light nuclei into somewhat heavier nuclei but these are still less massive than the iron-group nuclei characteristic of NSE. An accidental effect of the main nuclear reactions between the light nuclei in this scenario is the liberation of nucleons. If iron-group or heavier nuclei already exist in the system, they can capture these nucleons to produce new heavy nuclei. In this scenario, then, the system must have pre-existing seed nuclei in order to produce heavy elements. We call a process that requires pre-existing seed nuclei to make new elements a secondary process.

These are the only two ways nature can assemble heavy elements. We should not be surprised then that there are two major distributions of heavy nuclei-the $r$-process and the s-process distributions. We shall see that the r-nuclei in our Solar System likely formed in an environment that experienced a freezeout from equilibrium while the s-nuclei must have formed in an environment that was striving for, but never reached, NSE. The differing character of these scenarios results in the different character of the $\mathrm{r}$ - and s-process abundance distributions.

Once an abundance of heavy elements is available, nature may make modifications to it by exposing it to a flux of photons, neutrinos, or nucleons. Such events are probably responsible for the production of the majority of p-nuclei.

\section{THE r-PROCESS}

Nature does not endure sudden mutations without great violence.

François Rabelais, Gargantua

We turn now to a discussion of the r-process. We seek to understand in some detail how and where the r-process occurs. How the r-process occurs depends on whether it is a primary or secondary process. We will see that the evidence available to us today indicates that the r-process is a primary, freezeout from equilibrium process. As for where the r-process occurs, the rapid timescales associated with the r-process point to violent events such as supernovae or disruptions of neutron stars. Winds from nascent neutron stars are currently believed to be the most probable site. Our discussion will be brief. For more 
details, the reader should turn to the many excellent reviews in the literature (e.g. Hillebrandt 1978, Mathews \& Cowan 1990, Cowan et al 1991).

\subsection{The Primary r-Process}

Let us return to the notion of a freezeout from equilibrium. As a system in NSE expands and cools, the abundances shift to maintain NSE. We have seen that in doing so the entropy of the system moves from the baryons into the photons and leptons and thie abundance of heavier nuclei grows at the expense of free nucleons and light nuclei. Eventually the temperature of the system is too low or the abundance of reactants too small for certain reactions to go fast enough to maintain NSE. These reactions freeze out. The first reactions to freeze out are charged-particle reactions. Neutron-capture reactions can continue, however, because they are not impeded by a nuclear Coulomb barrier. The nuclei present at the time of the freezeout of the charged-particle reactions then eventually capture the remaining neutrons.

If we suppose that the system is quite neutron rich, many free neutrons should exist after freezeout of the charged-particle reactions. One might imagine that as we increase the neutron richness of the system, we simply have more neutron-rich isotopes of the nuclei present in the system. However, there is a limit to how neutron rich the nuclei can get. This is because the nuclei eventually encounter neutron drip, at which point the binding energy of the next neutron captured is negative. Once the nuclei reach neutron drip, they cannot contain any more neutrons. Thus, for sufficiently neutron-rich material, there may be many free neutrons at the time of charged-particle reaction freezeout. The system establishes an equilibrium between the neutron-capture $(n, \gamma)$ and neutron-disintegration $(\gamma, n)$ reactions. Beta decays then occur which increase the charge on the nucleus and allow further neutron capture. This phase of the freezeout from equilibrium in which only neutron-capture, neutron-disintegration, and beta-decay reactions occur is the r-process.

How neutron rich must material be to undergo an r-process? We know that the r-process produces uranium $(A=238)$ from seed nuclei $(A \approx 50-100)$; therefore, there must be roughly 100 free neutrons per seed nucleus at the time of charged-particle reaction freezeout. The required neutron richness thus depends on the abundance of seed nuclei which in turn depends on the entropy per baryon in the system.

If the entropy per baryon is less than or roughly $10 k$, NSE favors irongroup nuclei. These are the seed nuclei for the r-process once the chargedparticle reactions have frozen out. Because the r-process needs around 100 free neutrons per seed, a seed nucleus like ${ }^{78} \mathrm{Ni}$, typical in a low entropy, neutron-rich freezeout, requires a total neutron-to-proton $(\mathrm{n} / \mathrm{p})$ ratio of $(50+100) / 28 \approx 5.4$ or a $Y_{\mathrm{e}}=\mathrm{p} /(\mathrm{n}+\mathrm{p}) \approx 0.16$. This is quite neutron-rich material.

If the entropy per baryon is high $(\gtrsim 100 k)$, NSE favors ${ }^{4} \mathrm{He}$ at high tem- 
perature. As the temperature drops in the expanding material, NSE begins to favor iron-group nuclei. For large entropy per baryon, this occurs late in the expansion. At this late time, the two reaction sequences that begin the assembly of alpha particles into iron-group nuclei, ${ }^{4} \mathrm{He}+{ }^{4} \mathrm{He}+{ }^{4} \mathrm{He} \rightarrow{ }^{12} \mathrm{C}$ and ${ }^{4} \mathrm{He}+$ ${ }^{4} \mathrm{He}+\mathbf{n} \rightarrow{ }^{9} \mathrm{Be}$ followed by ${ }^{9} \mathrm{Be}+{ }^{4} \mathrm{He} \rightarrow{ }^{12} \mathrm{C}+\mathrm{n}$, may not be operating at a significant level or may even have frozen out. We note that these reaction sequences rely on three-body interactions which are highly sensitive to the density. The higher the entropy per baryon at a given temperature, the larger the photon-to-baryon ratio and the lower the density. A lower density results in slower three-body reaction rates. Thus the higher the entropy per baryon, the higher the temperature at which these three-body reactions freeze out and the lower the abundance of seed nuclei. Unlike the three-body reactions, alpha captures on ${ }^{12} \mathrm{C}$ and heavier nuclei occur rapidly and build up heavy nuclei. As the system falls out of equilibrium, there will be more alpha particles around than there would be in NSE. Some of these alpha particles capture on the iron-group nuclei present to make heavier nuclei $(A \approx 100)$. Eventually these reactions freeze out also, and the system is left with an abundance of free neutrons, seed nuclei, and many ${ }^{4} \mathrm{He}$ nuclei. At this point the free neutrons can capture on the seed nuclei, but not on the ${ }^{4} \mathrm{He}$ nuclei. In this way an r-process occurs.

The degree of neutron richness necessary for an r-process in a high entropy environment is less than in a low entropy environment. A typical composition for an entropy per baryon of $400 k$ and a neutron-to-proton ratio of $1.6\left(Y_{\mathrm{c}}=\right.$ 0.385 ) is $20 \%$ free neutrons by mass, $10 \%$ seed nuclei, and $70 \%$ alpha particles (Woosley \& Hoffman 1992). Suppose the seed nucleus is $Z=35, A=100$ (also typical), then the free neutron-seed nucleus ratio is 200 in the case: more than sufficient for an r-process. We thus see that the degree of neutron richness we need for a primary r-process directly depends on the entropy per baryon.

We have to this point discussed how a primary r-process would occur in the freezeout from NSE in low entropy and high entropy environments. The r-process might also occur in a very low entropy or zero-entropy environment. Matter inside a neutron star is extremely neutron rich and highly-degenerate. If the neutron star is more than a few hours old, it is cold $\left(T \lesssim 10^{9} \mathrm{~K}\right)$ on a nuclear energy scale (e.g. Baym \& Pethick 1979), implying that the entropy per baryon is quite low $(\lesssim 0.5 k)$. At densities below nuclear density $(\rho \approx 2 \times$ $\left.10^{14} \mathrm{~g} \mathrm{~cm}^{-3}\right)$ but above neutron-drip density $\left(\rho \approx 4 \times 10^{11} \mathrm{~g} \mathrm{~cm}^{-3}\right)$, extremely neutron-rich nuclei exist in strong equilibrium with degenerate neutrons and weak equilibrium with the degenerate electrons (Baym et al 1971). Above nuclear density, the material is comprised of free nucleons and electrons. Weak equilibrium forces the $Y_{\mathrm{e}}$ of the material to be of order 0.05 or less (Lattimer et al 1985, Lattimer \& Swesty 1991).

Only the strong gravity of the neutron star keeps such matter from exploding apart. If a piece of cold neutron-star matter were to escape from the neutron star 
into interstellar space, it would decompress. If this escape occurred without too much violence, there would not be a dramatic increase in the entropy. The material would remain cold. The material would consist of neutrons and nuclei, and as the material expanded, an r-process could occur (Lattimer et al 1977, Meyer 1989). In such a system, the only heating that would occur would be from the beta decays and nuclear fissions during the r-process, which are irreversible processes. Notice that such an r-process would not be a freezeout from NSE. The system was not in nuclear statistical equilibrium prior to expansion, and it would not attain NSE later in the expansion unless the beta decays and nuclear fissions that occur could drive the temperature up high enough, something that probably does not happen (Meyer 1989). On the other hand, this would be a freezeout from weak equilibrium. Thus, such an r-process would be primary because it is the formation of the neutron star that creates the seed nuclei and the weak equilibrium would erase the entire previous history of the nucleons. Moreover, material that began at densities above nuclear matter density would experience a phase transition from free nucleons into neutrons and nuclei during the expansion. In this case, the seed nuclei would form during the decompression.

Now that we understand how the r-process occurs in freezeout from equilibrium, we may consider the question of what astrophysical sites could give low, very low, or high entropy r-processes. Let us begin with low entropy sites. The earliest site considered for a low entropy r-process was at the mass cut of a type II supernova (Burbidge et al 1957, Cameron 1957). The mass cut is the boundary between the matter that escapes into space from the supernova and the matter that remains as part of the remnant neutron star. The first serious time-dependent calculations of such an r-process were made by Seeger et al (1965), although this work assumed constant temperature and neutron number density. Later calculations treated the r-process as dynamical, that is, with varying temperature and neutron density (Cameron et al 1970, Schramm 1973, Sato 1974, Kodama \& Takahashi 1975, Hillebrandt et al 1976, Hillebrandt 1978). While some of these workers found fairly good fits to the solar system r-process distribution, the models studied provided no natural way of explaining why the particular $\mathbf{r}$-process distribution we see should emerge from a supernova and, more seriously, why a supernova should eject only a small mass of r-process matter (see Section 3.3). It is also important to note that current type II supernova models do not yield $Y_{\mathrm{e}}$ as low as $0.1-0.2$ at the mass cut, as required by a low entropy r-process (e.g. Wilson \& Mayle 1993, Woosley et al 1994).

Due to the difficulties with the mass-cut site, astrophysicists turned to other supernova scenarios. In particular, workers sought other means of ejecting low entropy, neutron-rich r-process matter. Rotating stellar cores with (LeBlanc \& Wilson 1970, Meier et al 1976, Müller \& Hillebrandt 1979) and without magnetic fields (Symbalisty 1984, Symbalisty et al 1985) can eject some neutron- 
matter. It is not clear, however, that the supernova cores can attain the high rotation rates and magnetic fields required for such ejection. Moreover, there is no natural explanation for why the material has just the right conditions to make a solar system r-process distribution.

What about very low entropy r-processes? Lattimer \& Schramm (1974, 1976) considered the tidal disruption of a neutron star by a black hole. The ejected neutron-star matter could then undergo a very low entropy r-process (Lattimer et al 1977, Meyer 1989). Neutron star-neutron star collisions could also lead to the ejection of neutron-rich matter (Symbalisty \& Schramm 1982, Eichler et al 1989; see also Kochanek 1992 and Colpi et al 1989, 1991, 1993). If this material were not strongly disturbed during the collision, it would undergo a very low entropy r-process. It is likely in such an event, however, that the material would be shocked to entropies of order several $k$ per baryon, so that in fact a low entropy would probably ensue (Evans \& Mathews 1988). In any case, these sites suffer the two difficulties of uncertain occurrence rates and the lack of any natural reason a solar system r-process distribution should result.

This leaves the high entropy r-process. A promising site for such an r-process is in the neutrino-driven winds from nascent neutron stars. We consider this site in more detail in Section 3.4.

\subsection{The Secondary $r$-Process}

A system that does not achieve equilibrium may also produce r-process elements. As we have seen, as the bulk of the nuclei strive to reach the iron group, the reactions that carry them in this direction may release neutrons that then capture on pre-existing seed nuclei. If the number of neutrons released is sufficiently large, a solar system r-process abundance distribution may result. The nuclear reactions that would be the dominant producers of neutrons are $(\alpha, n)$ reactions, and in particular the reactions ${ }^{13} \mathrm{C}(\alpha, n){ }^{16} \mathrm{O},{ }^{22} \mathrm{Ne}(\alpha, n){ }^{25} \mathrm{Mg}$, and ${ }^{25} \mathrm{Mg}(\alpha, n){ }^{28} \mathrm{Si}$.

A crucial feature of a secondary r-process is that it does not achieve $(n, \gamma)-$ $(\gamma, n)$ equilibrium. Such equilibrium occurs when the flows from $(n, \gamma)$ and $(\gamma, n)$ reactions come into balance. The most abundant isotope of some element in such equilibrium is then the one for which the rate for that isotope $(Z, A)$ to capture a neutron is equal to the rate for the resultant isotope $(Z, A+1)$ to suffer a disintegration $(\gamma, n)$ reaction. The neutron-separation energy $S_{n}$, that is, the binding energy of the least tightly bound neutron, of this nucleus is, upon neglect of the $A$ dependence of nuclear partition functions (e.g. Sato 1974, see also Howard et al 1993, and Meyer 1994),

$$
S_{\mathrm{n}}(\mathrm{MeV})=\frac{T_{9}}{5.04}\left(34.08+1.5 \log _{10} T_{9}-1.5 \log _{10} n_{\mathrm{n}}\right)
$$

where $n_{\mathrm{n}}$ is the neutron number density in units of $\mathrm{cm}^{-3}$. In secondary r-process 
sites, the neutron number density is typically $\sim 10^{19} \mathrm{~cm}^{-3}$ and $T_{9} \sim 1$; thus, the dominant nuclei in $(n, \gamma)-(\gamma, n)$ equilibrium have $S_{\mathrm{n}} \approx 3.0 \mathrm{MeV}$. Figure 8 shows the neutron-separation energies for the isotopes of neodymium $(Z=60)$. We see that the dominant isotope in $(n, \gamma)-(\gamma, n)$ equilibrium should be the one with neutron number $N \approx 110$. The most neutron-rich beta-stable isotope of neodymium is ${ }^{150} \mathrm{Nd}(N=90)$. The neutron sources in a secondary r-process would have to supply and maintain some 20 neutrons per seed nucleus in order to establish $(n, \gamma)-(\gamma, n)$ equilibrium. This is something they cannot do.

Let us now consider possible secondary r-process sites. One possible site is the helium shell of an exploding massive star (Truran et al 1978, Thielemann et al 1979; see also Cowan et al 1980, 1983, 1985; Blake et al 1981; Klapdor et al 1981). As the supernova shock wave traverses this shell, it heats up the material to a temperature $T_{9} \approx 1$. Although this temperature is not high enough to force the material into NSE, it is high enough to drive material strongly in that direction. Among the main nuclear reactions that occur are $(\alpha, n)$ reactions that liberate neutrons which can then capture on the pre-existing seed nuclei. Truran et al (1978) found that neutron captures could modify an s-process seed abundance distribution into an r-process distribution. Later work indicated that a seed distribution that is enhanced with respect to the solar system heavyelement distribution is required to produce the solar $r$-nuclei. Such an enhanced distribution could result from s-processing prior to shock passage if protons were to mix down into the helium shell to make ${ }^{13} \mathrm{C}$ from the abundant ${ }^{12} \mathrm{C}$ / via

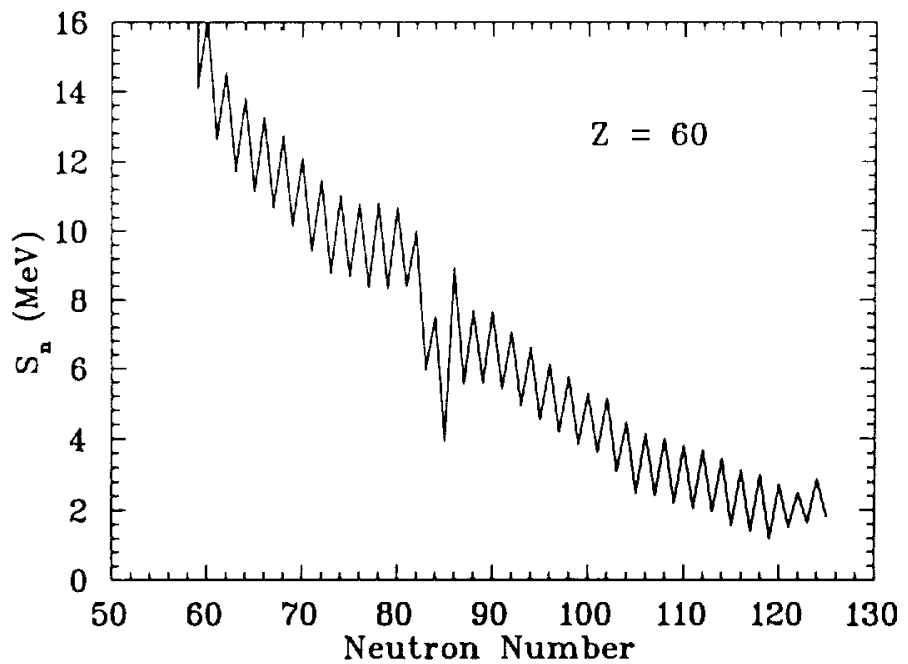

Figure 8 Neutron-separation energies for the isotopes of neodymium. The data are derived from Möller \& Nix (1988). 
$\left.{ }^{12} \mathrm{C}(p, \gamma){ }^{13} \mathrm{~N}\left(\beta^{+}\right){ }^{13} \mathrm{C}\right]$. It is apparent today, however, that the amount of ${ }^{13} \mathrm{C}$ required is unrealistically large (Cowan et al 1985, Cameron et al 1985).

Lee et al (1979) considered supernova shock passage through the carbon shell. Here the ${ }^{22} \mathrm{Ne}(\alpha, \gamma){ }^{25} \mathrm{Mg}$ reaction produces the neutrons. ${ }^{25} \mathrm{Mg}$ is also a neutron poison, however; it absorbs many of the liberated neutrons before they have a chance to capture on heavier seed nuclei. Wefel et al (1981) found that some heavy nuclei could be synthesized, but not the bulk of the r-nuclei.

Another intriguing secondary site is again the helium burning shell, but now the effects of neutrino inelastic scattering on ${ }^{4} \mathrm{He}$ nuclei are included. The neutrinos come from the cooling nascent neutron star resulting from the corecollapse event. These neutrinos spall neutrons from the ${ }^{4} \mathrm{He}$ (and other light) nuclei. The neutrons can then capture on the seed nuclei and drive an r-process (Epstein et al 1988). A detailed study of this " $v$-process" showed that some interesting nucleosynthesis may occur in such an event, but it could not have been a major contributor to the solar system r-process abundances (Woosley et al 1990).

All of the secondary r-process models studied to date have had profound difficulties which have rendered them implausible. Moreover, as shown in the next section, there are other reasons for favoring a primary over a secondary r-process. Nevertheless, the study of secondary r-process models has been valuable because they may have important applications to isotopic anomalies in meteorites (e.g. Clayton 1989, Howard et al 1992, Cameron et al 1993).

\subsection{Observational Constraints}

We have discussed how the r-process works in both primary and secondary scenarios and possible sites for the r-process. Let us turn now to the "observational" data to evaluate the plausibility of the proposed sites. These data will be astronomical, meteoritical, and nuclear.

We begin with the observations of old stars carried out by Sneden and collaborators (Sneden \& Parthasarathy 1983, Sneden \& Pilachowski 1985, Gilroy et al 1988; see also Wheeler et al 1989). These workers find that the elemental abundances, i.e. abundances as a function of $Z$, the proton number, of heavy elements in the atmospheres of these old stars match rather well the solar system r-process elemental abundances; s-process elemental abundances do not provide a good fit. One conclusion we may draw from this is that the r-process dominated the s-process at early times in our Galaxy. This would strongly suggest that the r-process is primary because a secondary r-process must produce $\mathrm{r}$-nuclei from an already abundant population of s-nuclei. Another important conclusion is that the r-process mechanism has remained essentially the same throughout the Galaxy's history. A caveat to both of these conclusions is that they are based on elemental abundances, whereas a truly definitive identification of the heavy nuclei in old stars as r-process in origin would require isotopic 
abundances, information that spectroscopy cannot yet generally give us. Nevertheless, the evidence for a primary r-process that has operated in the same fashion throughout the Galaxy's history is compelling.

The next item of evidence we may consider is the question of the timescale for heavy element formation. Mathews \& Cowan (1990) and Mathews et al (1992, 1993) have fit chemical evolution models to observations of the elemental abundances of europium (a mostly r-process element) and iron in the atmospheres of old stars. The only models that give good fits to the data are a primary r-process in type II and Ib (that is, core-collapse of a massive star) supernovae or a secondary r-process in which the neutron source itself is primary. An example of a primary neutron source in a secondary r-process event is the ${ }^{13} \mathrm{C}$ produced by mixing of protons down into the helium shell for supernova shock-induced helium or carbon burning. This is effectively a primary source because the star itself constructs the ${ }^{13} \mathrm{C}$ from its initial supply of protons and from ${ }^{12} \mathrm{C}$ made during helium burning. Another example is the neutrino-induced r-process. The neutrons in this source come mostly from inelastic scatterings of neutrinos on ${ }^{4} \mathrm{He}$ nuclei in the helium shell. The star itself produced almost all of the ${ }^{4} \mathrm{He}$ nuclei, so the neutron source is primary. Other proposed sites seemingly ruled out by these timescale arguments are: tidal disruptions of neutron stars by black holes, neutron star-neutron star collisions, a secondary r-process with a secondary neutron source such as ${ }^{22} \mathrm{Ne}$, neutron-star accretion disks (Hogan \& Applegate 1987), core helium flash in low-mass stars (Cowan et al 1982), and classical novae (Hoyle \& Clayton 1974).

Experimental nuclear physicists have also provided us with important clues about the r-process. In particular, K.-L. Kratz and collaborators have measured the beta-decay lifetimes of r-process "waiting pointing" nuclei on the $N=$ $A-Z=50$ and $N=82$ closed neutron shells (Kratz et al 1988, 1990). These nuclei have particularly strongly bound valence neutrons. Now the total abundance of the element $Z$ is a sum over the abundances of all of the isotopes of that element:

$$
Y_{Z}=\sum_{A} Y(Z, A)
$$

The average rate of beta-decay flow out of element $Z$ is

$$
\lambda_{Z}=\frac{\sum_{A} \lambda_{\beta}(Z, A) Y(Z, A)}{Y_{Z}},
$$

where $\lambda_{\beta}(Z, A)$ is the beta-decay rate of nucleus $(Z, A)$. The abundance $Y_{Z}$ decreases during the r-process by beta decay out of $Z$ into $Z+1$ and increases by beta decay into $Z$ from $Z-1$; thus,

$$
\frac{d Y_{Z}}{d t}=-\lambda_{Z} Y_{Z}+\lambda_{Z-1} Y_{Z-1}
$$


Where the r-process path crosses the $N=50$ and $N=82$ closed shells, a single isotope dominates the abundances because of the strong neutron binding. For these nuclei, $Y_{Z} \approx Y(Z, Z+50)$ for the $N=50$ closed neutron shell and $Y_{Z} \approx Y(Z, Z+82)$ for $N=82$. If the conditions for $(n, \gamma)-(\gamma, n)$ equilibrium hold for a long enough time, the system will achieve approximate steady beta flow such that $d Y_{Z} / d t \rightarrow 0$. In this case $\lambda_{Z} Y_{Z} \rightarrow$ constant. What is particularly significant is that the decay rates found by Kratz and co-workers times the appropriate abundances along the closed neutron shells as inferred directly from the solar system r-process data show that $\lambda_{Z} Y_{Z}$ is approximately constant for the $N=50$ and $N=82$ waiting-point nuclei. This is strong, albeit circumstantial, evidence that the r-process achieved the conditions of $(n, \gamma)-(\gamma, n)$ equilibrium and steady beta flow. This would indicate that the r-process is primary since a secondary r-process does not achieve $(n, \gamma)-(\gamma, n)$ equilibrium. Another important result found from this work is that the constant $\lambda_{Z} Y_{Z}$ for the $N=50$ closed neutron shell is different from that for the $N=82$ closed shell. The r-process did not achieve global steady beta flow (Kratz et al 1993). We interpret this to mean that the r-process abundance distribution actually results from a sum of components resulting from a varying set of $r$-process conditions - no single set of conditions gives rise to the solar system abundance curve. While this point was long appreciated by theoreticians (Seeger et al 1965, Kodama \& Takahashi 1975, Hillebrandt et al 1976), the work of Kratz and collaborators establishes this result directly from nuclear physics and meteoritic abundances. The actual r-process site in nature must naturally give rise to a varying set of r-process conditions.

The final observational constraint we consider is the mass of r-process material in the Galaxy. From meteorites and abundances in the Sun we can infer that the mass fraction of $\mathrm{r}$-nuclei in the Galaxy is $2 \times 10^{-7}$ (from the data in Anders $\&$ Grevesse 1989). If the mass of our Galaxy is $1.5 \times 10^{11} M_{\odot}$, there are some $10^{4} M_{\odot}$ of r-process material in our Galaxy. Now the rate of supernovae in our Galaxy is between $0.1 \mathrm{yr}^{-1}$ and $0.01 \mathrm{yr}^{-1}$ (e.g. Tammann 1982, Van den Bergh \& Tammann 1991) and the Galaxy is of order $10^{10}$ years old; therefore, there have been some $10^{8}$ to $10^{9}$ supernovae in our Galaxy's history. If each of these supernovae produced r-process material, we expect each supernova to make $10^{-5}$ to $10^{-4} M_{\odot}$ of $\mathrm{r}$-nuclei. This is a tiny fraction of the total mass ejected in a supernova and its smallness provides an important constraint on the site of the r-process.

From the astronomical and nuclear observations, we have found that the $r$ process is likely a primary event occurring in core-collapse supernovae. If the r-process comes from low entropy, neutron-rich material ejected from the core in a supernova, then we know from the discussion in Section 2.1 that we need material with $Y_{\mathrm{e}} \lesssim 0.2$ to make actinide nuclei. Such material must come from fairly deep in the core. Hillebrandt et al (1976) found that a supernova needs 
to eject of order $0.1 M_{\odot}$ of material to get $Y_{\mathrm{e}} \approx 0.1-0.2$ material out of the core. Such a large amount would overproduce the r-nuclei by a factor of $10^{3}$ if all supernovae ejected this much neutron-rich matter. It may be that only rare supernovae, such as those with high magnetic fields and rotation rates (LeBlanc \& Wilson 1970, Meier et al 1976, Müller \& Hillebrandt 1979, Symbalisty 1984, Symbalisty et al 1985), can eject neutron-rich matter. It remains to be seen, however, whether the high magnetic fields and/or rotation rates in these scenarios are indeed achieved in nature. In conclusion, we have no natural scenario for a low entropy primary r-process that yields $10^{-4} M_{\odot}$ of r-process material per event. Fortunately, we do have a high entropy r-process that gives this yield of $r$-nuclei per supernova. We turn to this scenario in the next section.

\subsection{The r-Process in Nascent Neutron Star Winds}

Woosley \& Hoffman (1992) proposed the winds from nascent neutron stars as the site of the r-process. In this section we discuss the physics of these winds. Only detailed models will allow us to determine whether these winds are indeed the site of the r-process. On the other hand, simple arguments will illustrate the basic features of these winds and why they are attractive as an r-process site.

A core-collapse (i.e. type II or Ib) supernova may leave a hot $(k T \approx 10 \mathrm{MeV})$ neutron star as a remnant (e.g. Bethe 1990). This neutron star cools by neutrino emission on a timescale of order 10 seconds. Salpeter \& Shapiro (1981) were among the first to consider the thermal evolution of such a young neutron star. They were able to show that, although the neutrino luminosity is always sub-Eddington, the photon luminosity can be super-Eddington. Thus, for sufficiently hot nascent neutron stars, there is a neutrino-driven wind.

Duncan et al (1986) repeated the arguments of Salpeter \& Shapiro and showed that a spherically symmetric, nascent neutron star of radius $10.6 \mathrm{~km}$ and mass $1.4 M_{\odot}$ has a super-Eddington photon luminosity if the neutron star temperature is $\gtrsim 0.4 \mathrm{MeV}$. Duncan et al also showed that the star cannot stabilize itself by changing its size or by transporting energy by convection. A wind must therefore blow from the surface of the neutron star. Moreover, Duncan et al showed that the mass loss rate in this wind is of order $10^{-5} M_{\odot} \mathrm{s}^{-1}$, which gives, if the wind lasts for the early cooling time of the nascent neutron star $(\sim 10 \mathrm{~s})$, about $10^{-4} M_{\odot}$. If this material forms r-process nuclei, the wind naturally gives the correct amount of r-process matter per supernova. [For more details on this wind, see Woosley et al (1994) and references therein.]

What about the entropy in the wind? The net heating of a matter element lifting off the surface of the neutron star is governed by the heating due to neutrino interactions with the wind material and cooling due to emission by the matter. The heating goes as $F_{\nu} \sigma\left\langle E_{v}\right\rangle$, where $\sigma$ is the neutrino-matter interaction cross section, $F_{v}$ is the number flux of neutrinos, and $\left\langle E_{v}\right\rangle$ is the average neutrino energy. $F_{v} \propto L_{v}\left\langle E_{v}\right\rangle^{-1} r^{-2}$ where $L_{v}$ is the neutrino luminosity and $r$ is the 
radial position of the matter element. $\sigma \propto\left\langle E_{\nu}\right\rangle^{2}$ (e.g. Tubbs \& Schramm 1975). The heating thus goes as $L_{v}\left\langle E_{v}\right\rangle^{2} r^{-2} .\left\langle E_{v}\right\rangle$ and $L_{v}$ fall off on the neutron-star cooling timescale of roughly 10 seconds. The matter elements move out on timescales faster than 10 seconds, so the heating rate falls off roughly as $r^{-2}$. The cooling goes as $T_{\mathrm{m}}^{6}$, where $T_{\mathrm{m}}$ is the local matter temperature. Above the surface of the neutron star, $T_{\mathrm{m}}^{6}$ falls off more steeply than $r^{-2}$. As the mass element lifts off the star, the initial heating is slow because the matter and neutrino temperatures are nearly equal. As the mass element moves out, the matter temperature $T_{\mathrm{m}}$ drops. Once the mass element passes the "gain radius," where the heating and cooling rates are equal, $T_{\mathrm{m}}$ is too low for the material to cool off as fast as it is heated (Bethe \& Wilson 1985). As heat is added, the entropy rises. In this way, the entropy can reach values of $100 k$ or more before neutrino interactions with the wind material freeze out. These are the values required for a high entropy r-process.

Note that the entropy in mass elements leaving the neutron star at late times will be larger than in mass elements leaving the star at early times. This is because most of the heating occurs fairly near the surface of the neutron star because the neutrino flux and hence the net heating rate falls off as $1 / r^{2}$. As a neutron star ages, it shrinks in radius [from $\sim 100 \mathrm{~km}$ at a few tenths of a second after core bounce to $\sim 10 \mathrm{~km}$ at several seconds after bounce in the models of Wilson \& Mayle (1993)]. The decrease in the initial $r$ from which the mass elements begin increases the heating rate more than the slow fall off in $L_{v}$ and $\left\langle E_{v}\right\rangle$ decrease it. The net heating and entropy in the later mass elements is thus larger.

The last question we must consider is the neutron richness of the wind material. $Y_{\mathrm{e}}$ is set in the wind by the reactions $\nu_{\mathrm{e}}+n \rightarrow p+e^{-}$and $\bar{\nu}_{\mathrm{e}}+p \rightarrow n+e^{+}$. If the fluxes and energies of the $\nu_{\mathrm{e}}$ and $\bar{\nu}_{\mathrm{e}}$ were equal, $Y_{\mathrm{e}}$ would be slightly larger than 0.5 because the mass of the proton is slightly less than that of the neutron. As a nascent neutron star cools, however, it becomes neutron rich. The opacity for $\nu_{\mathrm{e}}$ s becomes larger than that for $\bar{\nu}_{\mathrm{e}}$ s because of the reaction $v_{\mathrm{e}}+n \rightarrow p+e^{-}$. The $\bar{\nu}_{\mathrm{e}} \mathrm{s}$ thus have a longer mean free path and originate deeper in the neutron star. This means that they are more energetic than the $v_{\mathrm{e}} \mathrm{s}$. This necessarily drives the material neutron rich. At late times $(t \approx 10 \mathrm{~s}$ after core bounce) $\left\langle E_{\overline{\mathrm{i}}_{\mathrm{c}}}\right\rangle \approx 2\left\langle E_{\mathrm{v}_{\mathrm{c}}}\right\rangle$ which makes $Y_{\mathrm{e}} \approx 0.33$ (Qian et al 1993). This is certainly neutron rich enough for a high entropy r-process.

As a last point, let us re-emphasize that the entropy and $Y_{\mathrm{e}}$ in a mass element vary according to when that mass element lifts off the neutron star. Each mass element thus undergoes a somewhat different nucleosynthesis. The final r-process abundance distribution, however, is a sum of all of these different components. The $r$-process in nascent neutron star winds thus naturally satisfies the requirement imposed by the work of Kratz et al (1993). Notice that the wind dynamics and thermodynamics are completely determined by the mass, radius, and temperature of the neutron star. Any given neutron star probably passes 
through roughly the same sequence of temperatures and radii as a function of time as it cools. We thus expect to get essentially the same r-process out of every supernova. This satisfies our expectations from the observations of the r-process elements in old stars (see Section 3.3).

Does a solar system distribution naturally emerge in such a wind? Meyer et al (1992), using a schematic model based on output from Wilson \& Mayle (1993), found the resulting abundances matched the solar system distribution quite well (see also Howard et al 1993 and Takahashi et al 1994). A more detailed model, using mass element trajectories calculated directly in Wilson and Mayle's supernova code produced abundances that also agree well with the solar distribution (Woosley et al 1994). This latter model also gives the correct amount of r-process mass per supernova $\left(\sim 10^{-4} M_{\odot}\right)$. Confirmation of nascent neutron star winds as the site for the r-process will require a full survey of the nucleosynthesis in detailed, realistic wind models. Nevertheless, nascent neutron star winds seem extremely promising as the site for the r-process.

\section{THE s-PROCESS}

Must we, as Solon advises, always keep the goal in sight?

Aristotle, Nichomachean Ethics

The s-process is the other major nucleosynthetic process that assembles heavy elements. We know that the s-process path in the neutron number-proton number plane crosses the neutron closed shells at the valley of beta stability. This tells us that the s-process occurred in an environment with a much lower neutron density than the r-process. Also, the s-process occurred over a much longer time period.

In this section we seek to understand how the s-process occurs. We then turn to the question of s-process sites. Finally we consider constraints on those sites.

\subsection{The s-Process Mechanism}

Because of the neutron densities and timescales inferred for the s-process from the abundance peaks, we can infer that the s-process is not a freeze out from equilibrium. Instead, it is a neutron-capture process that occurs in a system striving to reach equilibrium, but falling short of its goal. The main reactions carrying the bulk of the nuclei towards the iron group can liberate neutrons. Pre-existing seed nuclei capture these neutrons and produce the s-nuclei. The s-process is clearly a secondary process.

The dominant reactions that can liberate neutrons are ${ }^{13} \mathrm{C}(\alpha, n){ }^{16} \mathrm{O}$ and ${ }^{22} \mathrm{Ne}(\alpha, n){ }^{25} \mathrm{Mg}$. In these reactions, the neutron-rich isotopes, ${ }^{13} \mathrm{C}$ and ${ }^{22} \mathrm{Ne}$ give up their excess neutrons to heavier nuclei. At this point, we may ask where 
these excess neutrons came from in the first place. The answer to this interesting question illustrates an important point about the overall nuclear evolution of the universe.

The abundances that emerge from the Big Bang are roughly $90 \%$ by number ${ }^{1} \mathrm{H}$ and $10 \%{ }^{4} \mathrm{He}$ (e.g. Walker et al 1991). This yields $Y_{\mathrm{e}}=0.88$. On the other hand, we may make the observation that ${ }^{1} \mathrm{H}$ and ${ }^{3} \mathrm{He}$ are the only proton-rich (that is, with proton number greater than neutron number) stable isotopes in nature. This means that in order for nature to put the nucleons in the universe into nuclei with the strongest binding energy per nucleon (iron-group nuclei), the $Y_{\mathrm{e}}$ of the universe must decrease.

Most of the decrease in $Y_{\mathrm{e}}$ comes from the weak decays in the $p$ - $p$ chains and the $\mathrm{CNO}$ cycle during hydrogen burning. These interactions drop $Y_{\mathrm{e}}$ from 0.88 to 0.5 in material that has completed hydrogen burning. ${ }^{4} \mathrm{He}$ itself does not have any excess neutrons, but some production of excess neutrons occurs in the CNO cycle due to reactions like ${ }^{12} \mathrm{C}(p, \gamma){ }^{13} \mathrm{~N}\left(\beta^{+}\right){ }^{13} \mathrm{C}$. The net result is the conversion of a free proton into an excess neutron, and a drop in $Y_{\mathrm{e}}$. The ${ }^{22} \mathrm{Ne}$ production builds up from abundant ${ }^{14} \mathrm{~N}$ produced in the $\mathrm{CNO}$ cycle. The sequence is ${ }^{14} \mathrm{~N}(\alpha, \gamma){ }^{18} \mathrm{~F}\left(\beta^{+}\right){ }^{18} \mathrm{O}(\alpha, \gamma){ }^{22} \mathrm{Ne}$. Here it is the fact that the only stable isotope of flourine is neutron rich that leads to a decrease in $\gamma_{e}$. We see that the excess neutrons in ${ }^{13} \mathrm{C}$ and ${ }^{22} \mathrm{Ne}$ are a consequence of the overall drive to decrease $Y_{\mathrm{e}}$ in stars. We must keep the goal of the nuclei in sight to understand where the excess neutrons come from that drive the s-process.

The first attempts to understand the details of the s-process led to the classical model. The neutron density is always low in the s-process (compared to the $\mathrm{r}$-process). If a nucleus is unstable to $\beta^{-}$decay following neutron capture in the s-process, it will almost always $\beta^{-}$decay to the first available stable isobar before it can capture another neutron. Thus, it generally suffices in s-process studies to follow only the abundances as a function of mass number, which only change by neutron capture. In this approximation, the rate of change of the abundance $N_{A}$ of nuclei with mass number $A$ is

$$
\frac{d N_{A}}{d t}=-n_{\mathrm{n}}\langle\sigma v\rangle_{A} N_{A}+n_{\mathrm{n}}\langle\sigma v\rangle_{A-1} N_{A-1} \text {, }
$$

where $n_{\mathrm{n}}$ is the neutron number density and $\langle\sigma v\rangle_{A}$ is the thermally averaged neutron-capture cross section for the stable isobar of mass number $A$. We can write $\langle\sigma v\rangle_{A}$ as $\sigma_{A} v_{\mathrm{T}}$, where $v_{\mathrm{T}}$ is the thermal velocity of neutrons and $\sigma_{A}$ is an average cross section, given in terms of $v_{\mathrm{T}}$. With the definition of the neutron exposure

$$
\tau=\int n_{\mathrm{n}} v_{\mathrm{T}} d t
$$

we find

$$
\frac{d N_{A}}{d \tau}=-\sigma_{A} N_{A}+\sigma_{A-1} N_{A-1} .
$$


Note that the neutron exposure $\tau$ is a fluence. It has units of inverse millibarns ( 1 barn $=10^{-24} \mathrm{~cm}^{2}$ ). Because it is a neutron flux integrated over time, it is an appropriate evolutionary parameter for the s-process. If the s-process achieves a steady state, then $d N_{A} / d \tau \rightarrow 0$ and $\sigma_{A} N_{A} \rightarrow$ constant.

Clayton et al (1961) were able to show that a single neutron exposure $\tau$ could not reproduce the solar system's abundance of s-only nuclei. Seeger et al (1965) showed that an exponential distribution of exposures, given by

$$
\rho(\tau)=\frac{f N_{56}}{\tau_{0}} e^{-\tau / \tau_{11}},
$$

where $f$ is a constant and $N_{56}$ is the initial abundance of ${ }^{56} \mathrm{Fe}$ seed, did reproduce the solar distribution of s-nuclei. For the distribution of exposures given in Equation (12), Clayton \& Ward (1974) found that for an exponential average of flows in the s-process

$$
\sigma_{A} N_{A}=f N_{56} \tau_{0} \prod_{A^{\prime}=56}^{A}\left[1+\left(\sigma_{A^{\prime}} \tau_{0}\right)^{-1}\right]^{-1} .
$$

A fit to the empirical $\sigma_{A} N_{A}$ for s-only nuclei then gives the quantities $f$ and $\tau_{0}$.

A complication to the above classical model is the branching that occurs at certain isotopes. Here it may be that the $\beta^{-}$decay rate is not considerably greater than the neutron-capture rate. In some cases the nucleus may $\beta^{-}$decay before neutron capture and in others it may neutron capture before suffering $\beta^{-}$decay. The assumptions leading to Equation (9) thus break down. Ward et al (1976) developed an analytic treatment of branching in the case of a time-independent neutron flux. For time-dependent neutron fluxes, it is necessary in general to solve a full network of nuclei numerically (e.g. Howard et al 1986). Since the s-process branchings will in general be temperature and neutron density dependent, s-nuclei branchings are important diagnostics of the environment in which the s-process occurred. We will see this in more detail in Section 4.3.

\section{$4.2 s$-Process Sites}

To obtain a good fit of the $\sigma N$ curve to the solar system s-process abundance distribution, three distinct exponential distributions of neutron exposures may be necessary (Clayton \& Rassbach 1967, Clayton \& Ward 1974). One exposure, with $\tau_{0} \approx 0.30 \mathrm{mb}^{-1}$, produces most of the nuclei in the mass range $90<$ $A<204$. This is the main component. Another exposure, with $\tau_{0} \approx 0.06$ $\mathrm{mb}^{-1}$ contributes to the $A \lesssim 90 \mathrm{~s}$-nuclei abundances. This weak component is required in order to explain the $\sigma N$ curve around $A \sim 90$. These two components indicate that two separate sites contributed to the abundance of solar s-nuclei. Finally, a strong component, with $\tau_{0} \approx 7.0 \mathrm{mb}^{-1}$, may be necessary to explain the abundances of the $A=204-209$ nuclei. One possible explanation 
of this component is that the distribution of exposures in the main component is not exactly exponential, but rather is higher than exponential at large $\tau$. There is probably no need for a separate site for the strong component of the s-process.

The weak s-process component likely comes from He burning in the cores of massive stars $\left(\gtrsim 15 M_{\odot}\right)$ (Truran \& Iben 1977, Lamb et al 1977), where the temperature is high enough for the ${ }^{22} \mathrm{Ne}(\alpha, n)^{25} \mathrm{Mg}$ reaction to produce a substantial amount of neutrons. These stars also have strong winds that eject this material into the interstellar medium. Recent work has confirmed the plausibility of this site (Arnett \& Thielemann 1985, Busso \& Gallino 1985, Prantzos et al 1987, Langer et al 1989, Raiteri et al 1991a, Baraffe et al 1992). Uncertainties in the ${ }^{22} \mathrm{Ne}(\alpha, n)^{25} \mathrm{Mg}$ and ${ }^{22} \mathrm{Ne}(\alpha, \gamma)^{26} \mathrm{Mg}$ reaction rates prevent us from predicting the neutron exposure in these models to high accuracy. Recent results on these rates may indicate that the s-process is somewhat more robust in this site than previously thought (e.g. Baraffe \& El Eid 1994). This may complicate the separation of the $A \lesssim 90$ s-nuclei into those coming from the weak and main components.

Some s-processing may also occur in core carbon burning or shell helium burning in massive stars. This has been studied by Arcoragi et al (1991) and Raiteri et al (1991b). The results indicate that this processing does not contribute in a significant way to the weak component.

The main component of the s-process is likely to occur in the helium-burning shell in asymptotic giant branch (AGB) stars (Weigert 1966, Schwarzchild \& Härm 1967, Ulrich 1973). The structure of such a star is an inert carbon-oxygen core, on top of which lies a convective helium-burning shell. On top of this helium-burning shell is the hydrogen-rich envelope, which itself is convective. The original idea was that the convective helium shell might reach out far enough into the hydrogen-rich envelope that protons and ${ }^{12} \mathrm{C}$ (the result of helium burning) could mix and produce ${ }^{13} \mathrm{C}$, as discussed in Section 4.1 . The ${ }^{13} \mathrm{C}$ would then be the source of neutrons for the s-process. [The current picure is that convection does not provide the mixing, but that protons reach down into the carbon-rich shell by diffusion or semiconvection (see below).]

An attractive feature of this model is the fact that the helium burning occurs in pulses. Between pulses, hydrogen burns quiescently in a thin shell. Once the supply of helium from the hydrogen burning builds up, a helium-burning pulse occurs. The energy liberated expands the star and shuts off the hydrogen burning. After the pulse has occurred, the star settles down again and begins hydrogen-shell burning anew. Pulses last of order tens of years while the interpulse periods are of order thousands of years. The significance for the s-process is that there is an overlap of mass zones experiencing successive helium-buring pulses. Ulrich (1973) was able to show that the mixing and burning sequence could naturally give rise to an exponential distribution of neutron exposures. Alternating overlap of convection zones can carry the newly 
produced s-nuclei into the envelope (the so-called "third dredge up"). These nuclei would then find their way into the interstellar medium via winds or by the ejection of the atmosphere in a planetary nebula phase.

This nice model for the s-process suffered a setback when Iben showed that an entropy barrier prohibited mixing of protons into the helium shell (Iben 1975a,b, 1976). It was then proposed instead that ${ }^{22} \mathrm{Ne}(\alpha, n){ }^{25} \mathrm{Mg}$ be the source (e.g. Iben $\&$ Renzini 1983). The helium core grows by accreting the ashes of the hydrogen-burning shell. The products of CNO burning are ${ }^{4} \mathrm{He}$ and ${ }^{14} \mathrm{~N}$ in that shell, which combine to give ${ }^{22} \mathrm{Ne}$ early in helium burning, as discussed in Section 4.1. The ${ }^{22} \mathrm{Ne}(\alpha, n)^{25} \mathrm{Mg}$ reaction then drives the s-process. The pulse and mixing that occurs gives an exponential distribution of neutron exposures. This model has some difficulties, however. Basically, the shell flashes in most AGB stars are not hot enough to liberate most of the ${ }^{22} \mathrm{Ne}$ neutrons, and the massive ABG stars that are hot enough are too rare. This has led workers to consider alternative neutron sources in low-mass AGB stars $\left(M<3 M_{\odot}\right)$.

In low-mass AGB stars, the temperature is too low in the helium-burning shell for the ${ }^{22} \mathrm{Ne}(\alpha, n)^{25} \mathrm{Mg}$ reaction to be the major source of neutrons. Iben \& Renzini (1982) argued, however, that, despite the entropy barrier to convection, semiconvection or diffusion could cause the mixing of protons with ${ }^{12} \mathrm{C}$ in the interpulse period. This produces pockets of ${ }^{13} \mathrm{C}$ atop the He zones which can liberate neutrons during convective ingestion by the next pulse. Recent work indicates that this is a promising site for the s-process (Gallino et al 1988; Boothroyd \& Sackmann 1988a,b,c,d; Hollowell \& Iben 1988; Käppeler et al 1990). In particular, these models seem to give a good fit to the main component of the solar $\sigma N$ curve (e.g. Käppeler et al 1990). We must note that these s-process calculations are post-processing calculations, which means that the neutron density is a parameterized quantity. Even more serious is the lack of a demonstrated occurrence of the needed ${ }^{13} \mathrm{C}$-rich pocket, which is therefore taken on faith at the present time. It remains to be seen whether the good agreement with the solar s-process abundances will hold up when the s-process calculations are directly coupled to complete stellar models. Such coupled calculations may be available in the not-too-distant future. It will also be important to include the effects of energy generation by all the nuclear reactions on the stellar structure (Bazan \& Lattanzio 1993).

\subsection{Constraints on s-Process Sites}

What constraints can help to evaluate the proposed sites discussed in the previous section? s-process branchings are the first important constraints. The likelihood that a beta-unstable nucleus in the s-process beta decays depends on the rate of beta decay compared to the rate of neutron capture. Evidence for branching provides information about these rates. In particular, with knowledge of the beta-decay rate from laboratory experiments, the degree of branching 
constrains the neutron capture rate $n_{\mathrm{n}}\langle\sigma v\rangle$. Then knowledge of $\langle\sigma v\rangle$ from the laboratory constrains $n_{\mathrm{n}}$, the neutron number density during the s-process. On the other hand, if the beta-decay rate is temperature sensitive (e.g. Takahashi \& Yokoi 1987), branching data yield constraints on the temperature during the s-process. Branching data may also yield constraints on the mass density during the s-process through electron capture rates. Finally, branching data can constrain the duration of the neutron pulses (Ward \& Newman 1978). If the pulse period were much shorter than the lifetime of the branching point isotope, there would be no branching. Pulses that were too long in duration would allow too much neutron capture.

What do we find for the s-process in nature? For the main component of the s-process, the isotopes ${ }^{134} \mathrm{Cs},{ }^{148} \mathrm{Pm},{ }^{151} \mathrm{Sm},{ }^{154} \mathrm{Eu},{ }^{170} \mathrm{Yb}$, and ${ }^{185} \mathrm{~W}$ are branch-point isotopes with potential as diagnostics of the temperatures and neutron densities prevailing during the s-process. Beer et al (1984) used ${ }^{151} \mathrm{Sm}$, ${ }^{170} \mathrm{Yb}$, and ${ }^{185} \mathrm{~W}$ to find limits on the neutron number density and temperature. Uncertainty in the population of the $137 \mathrm{keV}$ isomeric state in ${ }^{148} \mathrm{Pm}$ during the s-process makes conclusions from this isotope difficult. Uncertainties in cross sections and abundances limit the usefulness of ${ }^{134} \mathrm{Cs}$ and ${ }^{154} \mathrm{Eu}$.

As for the mass density, Yokoi \& Takahashi (1983) noticed that ${ }^{163}$ Dy could beta decay in stars, even though it is stable on Earth. In stars, the ${ }^{163}$ Dy atom is ionized so that in fact the daughter atom ${ }^{163} \mathrm{Ho}$ would be at slightly lower mass. ${ }^{163}$ Ho then could either capture a neutron or electron capture back to ${ }^{163}$ Dy. The electron capture rate depends on the density of electrons, which in turn depends on the mass density. Beer et al (1985) were able to constrain the mass density in the s-process in this way.

Finally, Beer \& Macklin (1988) studied ${ }^{151} \mathrm{Sm}$ in order to determine a lower limit to the duration of the neutron pulse in the s-process. Studies of ${ }^{86} \mathrm{Kr}$ may give an upper limit to the pulse duration (Beer \& Macklin 1989). Unfortunately the weak component in this region introduces ambiguities into such an analysis.

The net results of branching studies in the context of the classical model give a temperature for the main component of $2.8-3.9 \times 10^{8} \mathrm{~K}$, a neutron density of $2.3-4.5 \times 10^{8} \mathrm{~cm}^{-3}$, a mass density of $2.6-13 \times 10^{3} \mathrm{~g} \mathrm{~cm}^{-3}$, and a pulse duration of greater than 3 years (Käppeler et al 1989). These numbers agree reasonably well with those expected from stellar models. A similar analysis for the weak component yields a temperature of $1.8-3.0 \times 10^{8} \mathrm{~K}$ and a neutron density of $0.8-1.9 \times 10^{8} \mathrm{~cm}^{-3}$ (Käppeler et al 1989).

The relatively high temperatures found in this analysis for the main component suggest that ${ }^{22} \mathrm{Ne}(\alpha, n){ }^{25} \mathrm{Mg}$ is the neutron source for the s-process. Howard et al (1986) studied the s-process nucleosynthesis with this neutron source. They obtained poor fits to the solar $\sigma N$ curve when they used parameters derived from stellar models. In particular, the average neutron density during the pulses was too high to reproduce the correct branchings. Busso et 
al (1988) have confirmed these results. On the other hand, the high temperature ${ }^{22} \mathrm{Ne}$ source may simply be the last hot part of the neutron burst that was primarily from ${ }^{13} \mathrm{C}$ at lower temperature (see below).

Let us consider now the evidence from observations of stars. It was the observation of technetium in certain red giant stars (Merrill 1952) that showed that stars do indeed synthesize elements and led Cameron (1955) to work out many of the details of the s-process. Since all isotopes of Tc are unstable, any Tc present in the surface of a star must have been synthesized in the interior of the star by the s-process and then dredged up to the surface. Recent observations show that red giant stars in the solar neighborhood that do have s-process abundance enhancements in their atmospheres do not show the accompanying enhancements of ${ }^{25} \mathrm{Mg}$ and ${ }^{26} \mathrm{Mg}$ that one would expect from alpha capture on ${ }^{22} \mathrm{Ne}$ (e.g. Smith \& Lambert 1986, McWilliam \& Lambert 1988). In addition, observations of $\mathrm{Rb}$ and ${ }^{96} \mathrm{Zr}$ constrain s-process branching at ${ }^{85} \mathrm{Kr}$ and ${ }^{95} \mathrm{Zr}$. Astronomers find that the s-process occurring in the interiors of the stars observed must be happening at low neutron densities $\left(n_{\mathrm{n}} \lesssim 10^{9} \mathrm{~cm}^{-3}\right)$, not the high neutron densities characteristic of the ${ }^{22} \mathrm{Ne}(\alpha, n){ }^{25} \mathrm{Mg}$ reaction (e.g. Lambert 1993).

From this evidence, it appears that ${ }^{13} \mathrm{C}$ is more promising as the source of s-process neutrons, indicating that low-mass AGB stars are probably the site of the s-process. Such stars give a low temperature s-process $\left(\sim 1.5 \times 10^{8} \mathrm{~K}\right)$ which would seem to contradict the higher temperatures found from the analysis of the s-process branchings in the classical model $\left(T=2.8-3.9 \times 10^{8} \mathrm{~K}\right)$ discussed above. In the low-mass AGB star s-process calculations that do show good agreement with solar abundances (e.g. Gallino et al 1988, Käppeler et al 1990), there are two bursts of neutrons per pulse: a strong burst due to the ${ }^{13} \mathrm{C}(\alpha, n){ }^{16} \mathrm{C}$ reaction at $T \sim 1.5 \times 10^{8} \mathrm{~K}$, and a second, weaker one, due to the ${ }^{22} \mathrm{Ne}(\alpha, n)^{25} \mathrm{Mg}$ reaction. This weaker burst occurs when the helium shell contracts following the first burst and heats to a temperature of $T \sim 3 \times 10^{8} \mathrm{~K}$. It resets the branch-point thermometers to this higher temperature, in agreement with the analysis from the classical model.

More evidence for ${ }^{13} \mathrm{C}$ as the dominant source for neutrons in the s-process comes from studies of galactic abundance evolution. Mathews et al $(1992,1993)$ studied the evolution of the $\mathrm{Ba} / \mathrm{Fe}$ ratio in our Galaxy. Ba is predominantly an s-process element and hence must be secondary (i.e. made from initial $\mathrm{Fe}$ ). Mathews et al found that only an s-process behaving as a primary process fit well the observations of $\mathrm{Ba}$ abundances in the atmospheres of old stars. ${ }^{13} \mathrm{C}$ is a primary neutron source, as discussed in Section $3.3 .{ }^{22} \mathrm{Ne}$ is secondary because it must be built up from pre-existing CNO nuclei. The Fe seeds are of course secondary. Clayton (1988a) described how the secondary s-process with the ${ }^{13} \mathrm{C}$ neutron source is able to mimic primary nucleosynthesis. The idea here is that while the galactic abundance of Fe seed for the s-process grows with time, so does the abundance of s-process neutron poisons. 
A final point of increasing relevance is the new information from pre-solar $\mathrm{SiC}$ grains found in the Murray and Murchison meteorites. These grains are carriers of isotopic anomalies in s-process isotopes (Srinivasan \& Anders 1978, Tang \& Anders 1988). In addition, these grains are anomalous in their Si and C (Zinner et al 1987, Anders \& Zinner 1993). It appears that these grains have condensed in carbon-star atmospheres, which are s-process enriched and have variable ${ }^{13} \mathrm{C}$-rich compositions (Lambert et al 1986). As surviving stardust, the grains are almost pure endmembers in the "cosmic chemical memory" theory for interpreting isotopic anomalies in solar system samples (Clayton 1978, 1982). From studies of trace s-isotopes in these grains (Ott \& Begemann $1990 \mathrm{a}, \mathrm{b} ; \mathrm{Zin}$ ner et al 1991; Richter et al 1992; see Anders \& Zinner 1993 for a review) the abundance ratios only fit if the grains come from low-mass AGB stars (Gallino et al 1990). A vexing problem with this idea, however, is that such stars cannot explain the anomalous $\mathrm{Si}$ isotopes (a major constituent of the grains). One suggested answer is higher mass $\mathrm{AGB}$ stars, in which burning of $\mathrm{Mg}$ isotopes in late pulses resets the ratio of ${ }^{29} \mathrm{Si} /{ }^{30} \mathrm{Si}$ (Brown \& Clayton 1992). Galactic abundance evolution of Si isotopes may also hold the key (Clayton 1988b, Gallino et al 1994). Alternatively, some other site may be responsible for these grains (e.g. Arnould \& Howard 1993). These tiny, sturdy grains have traveled from afar carrying important messages about the s-process which have yet to be deciphered.

In summary, low-mass AGB stars are at present the most promising site for the main component of the s-process. Confirmation of this site will require continued interplay of nuclear physics, meteoritics, stellar evolution and structure theory, nucleosynthesis theory, galactic abundance evolution theory, and stellar astronomy. Many people will be busy for quite some time to come!

\section{THE p-PROCESS}

... and the elements shall melt with fervent heat ...

II Peter 3:10

We turn finally to the p-nuclei. These are the 35 nuclei bypassed by the $r-$ and s-processes. As we see from Figure 1, except for the light p-nuclei $\left({ }^{92} \mathrm{Mo}\right.$, ${ }^{94} \mathrm{Mo},{ }^{96} \mathrm{Ru},{ }^{98} \mathrm{Ru}$ ), the abundances of $\mathrm{p}$-nuclei are considerably less than those of their $\mathrm{r}$ - and s-nuclei counterparts. Furthermore, the p-process abundance distribution shows interesting structure with peaks at ${ }^{92} \mathrm{Mo}$ and ${ }^{144} \mathrm{Sm}$. These are important clues for determining where the p-process occurs.

It is probably wrong to think that the p-process occurs in a single site. We can imagine many astrophysical settings where conditions are right to modify a pre-existing supply of $r$ - and $s$-nuclei to form $p$-nuclei. The relevant question is really what site contributes the bulk of the p-nuclei. For more details on the p-process, the reader should consult the excellent review by Lambert (1992). 


\subsection{General Considerations}

It is impossible to produce p-nuclei by neutron capture. How then can nature make these nuclei? The first possibility that suggests itself is proton capture. It may be that in the course of the evolution of some system striving to reach NSE, protons are liberated which can capture on pre-existing seed nuclei to make p-nuclei. Alternatively it may be that in a freezeout from proton-rich NSE, free protons could capture on seed iron-group nuclei. $\beta^{+}$reactions could allow further capture of protons to higher mass.

We can explore the conditions required for such p-processes with the help of Figures 9 and 10. Figure 9 shows the timescale for capture of a proton [a $(p, \gamma)$ reaction] by the most proton-rich beta-stable isotope of each element at the fixed temperature of $T_{9}=1$ for different values of the proton mass density $\rho Y_{p}$. The timescales for a proton-disintegration $(\gamma, p)$ reaction or for a neutron-disintegration $(\gamma, n)$ reaction for these isotopes are all greater than $10^{10}$ seconds. Note that for a site with a proton mass density $\rho Y_{\mathrm{p}}$ of $1 \mathrm{~g} \mathrm{~cm}^{-3}$, it would take ${ }^{92} \mathrm{Mo}(Z=42)$ about $10^{4} \mathrm{~s}$ to capture a proton. Capture of protons on higher-charge isotopes would take even longer. The timescale for proton capture decreases if the setting has a higher density of protons available. For example, for $\rho Y_{\mathrm{p}}=10^{3} \mathrm{~g} \mathrm{~cm}^{-3}$, the timescale for capture of protons on ${ }^{92} \mathrm{Mo}$

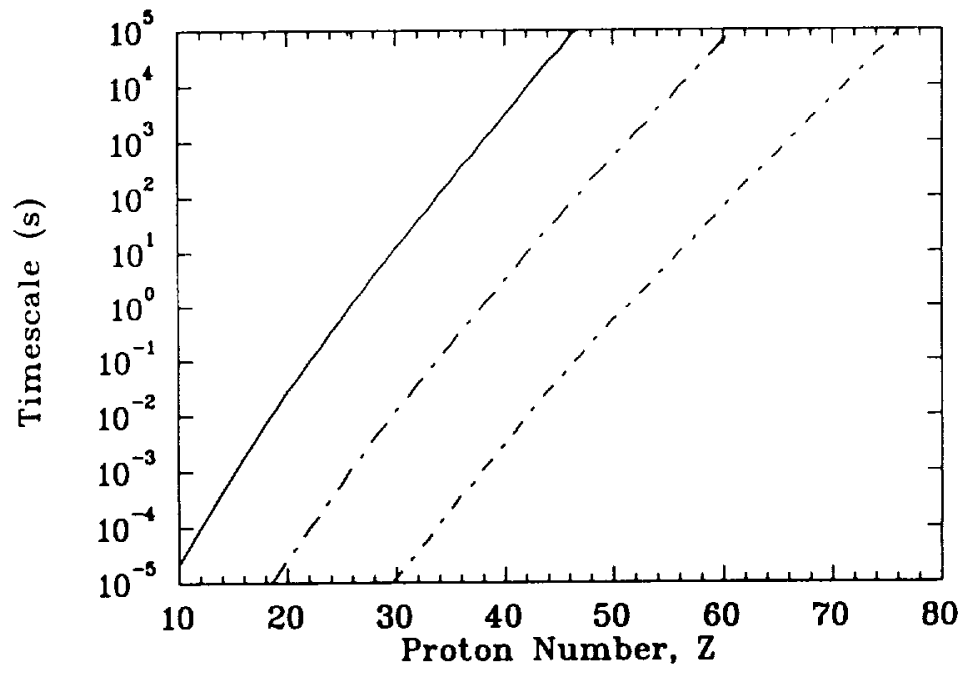

Figure 9 Timescales for proton capture on the most proton-rich isotope of each element at the fixed temperature of $T_{9}=1$. The curves are for mass densities in protons of $\rho Y_{\mathrm{p}}=1 \mathrm{~g} \mathrm{~cm}^{-3}$ (solid curve), $10^{3} \mathrm{~g} \mathrm{~cm}^{-3}$ (long dashed-dotted curve), and $10^{6} \mathrm{~g} \mathrm{~cm}^{-3}$ (short dashed-dotted curve). The rates are computed from expressions in Woosley et al (1975). 


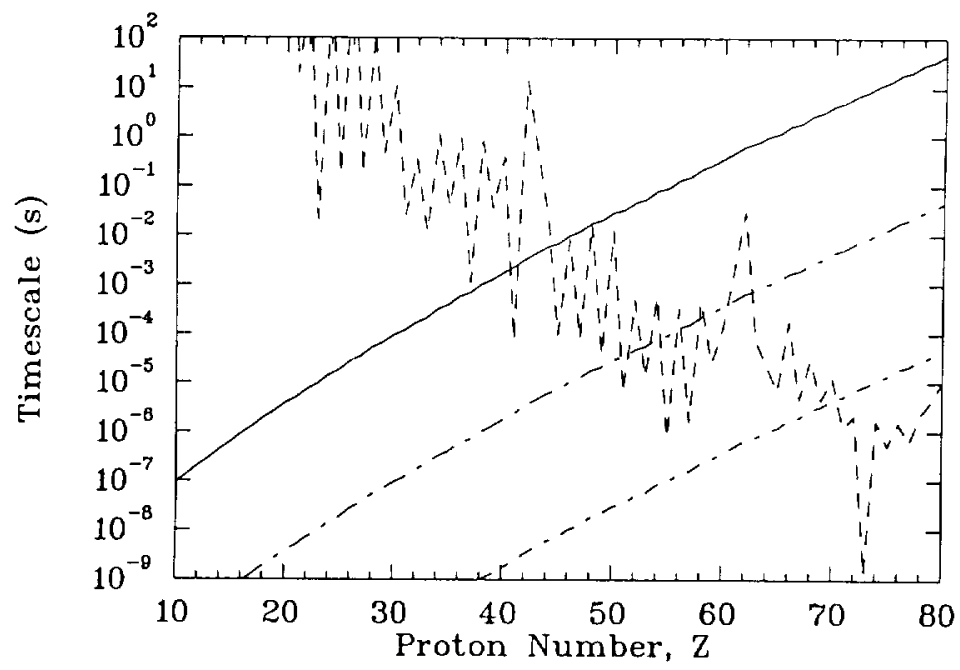

Figure 10 Same as Figure 9 but for $T_{y}=3$. The short-dashed jagged curve shows the timescale for $(\gamma, n)$ reactions on these nuclei. The $(\gamma, n)$ rates are computed from neutron-capture cross sections in Woosley \& Hoffman or Cowan et al (1991) and neutron-separation energies are derived from Möller \& Nix (1988).

would be about $10 \mathrm{~s}$ while for $\rho Y_{\mathrm{p}}=10^{6} \mathrm{~g} \mathrm{~cm}^{-3}$ it would be about $10^{-2} \mathrm{~s}$. If an astrophysical site could maintain a mass density in protons of $10^{6} \mathrm{~g} \mathrm{~cm}^{-3}$ for $10^{5} \mathrm{~s}$ at $T_{9}=1$, the proton-rich isotopes of all elements up to platinum $(Z=78)$ could capture a proton. The question for finding the p-process site is whether such conditions are possible. It is unlikely.

The proton-capture rates also increase with increasing temperature because the reactants have a higher relative kinetic energy compared to the Coulomb barrier than at lower temperature. This leads us to ask what happens to the timescales if we increase the temperature. We see the effect in Figure 10 for $T_{9}=3$. The timescale for proton capture does indeed decrease, but so does the timescale for a $(\gamma, n)$ reaction. For $\rho Y_{\mathrm{p}}=1 \mathrm{~g} \mathrm{~cm}^{-3}$, the timescale for a $(\gamma, n)$ reaction is less than that for a $(p, \gamma)$ reaction at $Z \approx 40$. This means that it is more likely under these conditions for a proton-rich nucleus to suffer a $(\gamma, n)$ reaction than to capture a proton. Of course this makes sense from our discussion in Section 2. If the system is evolving towards NSE, nuclei more massive than the nucleus with the highest binding energy per nucleon will tend to disintegrate nucleons to increase the number of macroscopic states available to the system.

For higher proton mass densities, the $(\gamma, n)$ reactions do not dominate the proton captures until higher nuclear charge: $Z \approx 40-50$ for $\rho Y_{\mathrm{p}}=10^{3} \mathrm{~g} \mathrm{~cm}^{3}$ and $Z \approx 70$ for $\rho Y_{\mathrm{p}}=10^{6} \mathrm{~g} \mathrm{~cm}^{-3}$. One might imagine that the system could 
then produce p-nuclei at least up to ytterbium $(Z=70)$ under such high temperature and high density conditions. Such conditions are extremely difficult for nature to achieve, however. The dilemma for making p-nuclei is clear. If nature is to make these nuclei by proton capture at low temperature where the flow will not be impeded by disintegrations, a large supply of protons must be available for a long time. If the proton capture process is to occur at higher temperature where the capture timescales are shorter, disintegration reactions will dominate the flow and prevent capture to higher mass.

The escape from this dilemma is the realization that proton captures need not make most of the p-nuclei. The various disintegration reactions do the job. In particular, if pre-existing $\mathbf{r}$ - and s-nuclei are exposed to high temperature, nuclear reactions will occur and tend to drive the abundances toward NSE. The first reactions to occur are the $(\gamma, n)$ reations which produce quite protonrich nuclei. Once the nuclei become sufficiently proton rich, they then begin a $(\gamma, p)$ and $(\gamma, \alpha)$ cascade. In this way the nuclei "melt" towards iron. If the high temperature drops off quickly enough, the system does not reach NSE and the melting will be incomplete, leaving an abundance of proton-rich heavy nucleithe p-nuclei. Where the disintegration flow crosses the $N=50$ and $N=82$ closed neutron shells, the disintegration timescales become large because of the particularly strong binding energies. This is apparent in Figure 10 as the peaks in the $(\gamma, n)$ timescales at the closed shell nuclei ${ }^{92} \mathrm{Mo}(Z=42)$ and ${ }^{144} \mathrm{Sm}$ $(Z=62)$. Because of the long disintegration timescales, abundances build up at these nuclei. In this way we can explain the peaks in the solar system's p-process abundance distribution. As a final note, the extremely short $(\gamma, n)$ timescale for ${ }^{180} \mathrm{Ta}(Z=73$ in Figure 10 ) explains why this fragile species is the rarest stable isotope in nature.

\section{2 p-Process Sites}

The early papers on the p-process considered the proton capture mechanism (Burbidge et al 1957, Ito 1961, Macklin 1970, Truran \& Cameron 1972, Audouze \& Truran 1975). The site was imagined to be the hydrogen-rich envelope in massive stars undergoing a supernova explosion. The supernova shock passing through this region would heat up the material and proton capture reactions would produce the p-nuclei. However, the densities, temperatures, and timescales required are unrealistic for the hydrogen-rich envelope (see, for example, the discussion in Woosley \& Howard 1978).

Arnould (1976) computed the p-process in the hydrostatic oxygen burning phase in stars. The timescales are longer in this site than in the supernova site and would allow for more proton capture. In this site, temperatures were high enough for disintegrations [especially $(\gamma, n)$ reactions] to be important. A major challenge for this model is to eject the new p-nuclei without significantly modifying their abundances during the subsequent supernova explosion. 
Woosley \& Howard (1978) computed the p-process in the O/Ne shell in type II, that is, core-collapse, supernovae. The supernova shock heats up this shell and causes the partial melting of the nuclei. In this model, only disintegrations are important, hence the alternative name "gamma-process" (see Rayet et al 1990, Prantzos et al 1990, Rayet et al 1992 for extensions of this model). This model successfully reproduced most of the p-nuclei in their solar system proportions, although it seriously underproduced the light p-nuclei. As in the hydrostatic oxygen burning model, it was necessary to superimpose several abundance distributions to get a realistic distribution of p-nuclei. Type II supernovae should naturally give a distribution of conditions depending on the layers of the proto-SN considered that would naturally give a distribution of abundances. The inner regions of the $\mathrm{O} / \mathrm{Ne}$ shell will achieve the highest temperatures and thus get closest to NSE. These regions make the lighter p-nuclei. Outer regions produce the heavier p-nuclei because the "melting" is less complete. Prantzos et al (1990) computed the p-process abundance distribution for a specific model of supernova 1987A and found that a distribution of conditions naturally arose and gave a solar system p-process abundance distribution, except for underproduction of light p-nuclei.

The problems with the underproduction of the light p-nuclei in the gammaprocess led Howard et al (1991) to consider the production of p-nuclei in the outermost layers of a carbon-oxygen white dwarf star suffering a type Ia supernova explosion. In this model, s-processing prior to the explosion built up the abundances of $A \approx 90$ nuclei. The high density during the explosion then allowed proton capture reactions to produce many of the light p-nuclei while the normal gamma-process made the heavier p-nuclei. Later calculations using the realistic type Ia models of Khoklov (1990) have not been as successful in producing the light p-nuclei (Howard \& Meyer 1992). More studies of this promising site are required.

Some p-nuclei may also be produced in spallation reactions. Most notably, neutrinos may spall neutrons from heavy nuclei during type II supernovae to make p-nuclei. Such a process is only likely to produce significant amounts of the rarest p-nuclei such as ${ }^{138} \mathrm{La}$ and ${ }^{180} \mathrm{Ta}$ (Woosley et al 1990).

\subsection{Some Constraints on p-Process Models}

As mentioned above, the p-process probably occurs in several places in nature. Any astrophysical setting in which high temperatures but sufficiently short timescales lead to incomplete melting of heavy nuclei can produce p-nuclei. What we really seek is the site that produces the bulk of the p-nuclei. We can attempt to analyze this requirement by means of overproduction factors.

The overproduction factor of an isotope in the product material of some nucleosynthetic process is the ratio of its mass fraction in that material to its mass fraction in the solar system. In order for the process to be responsible 
for the production of the bulk of the solar system's supply of a given isotope, that isotope must have the largest overproduction factor in the product material. If the process is to be responsible for the bulk of the solar system's supply of two or more isotopes, those isotopes must all have comparably large overproduction factors.

Prantzos et al (1990) found an average overproduction factor of 0.96 for p-nuclei in $15 M_{\odot}$ of ejecta in their model for SN 1987A. The overproduction factor for ${ }^{16} \mathrm{O}$ in the same model was 11.5 (Thielemann et al 1990). Since type II supernovae made most of the solar system's ${ }^{16} \mathrm{O}$, this would indicate that the type II supernova site could not be responsible for the production of the bulk of the solar system p-process elements. Prantzos et al argue, however, that there are extenuating factors. First, the SN 1987A model used Large Magallenic Cloud metallicities, which are lower than those in our Galaxy. Milky Way metallicities could give enhanced seed abundances and thus higher p-process overproduction factors. Also, it may be that p-nuclei production relative to oxygen could be higher in higher mass stars. Surveys over a range of star masses, such as that of Arnould et al (1992), will be important for understanding the contribution of type II supernovae to the solar system p-process abundances.

As for the type Ia model, Howard et al (1991) noted that besides possibly producing p-nuclei, these supernovae made most of the solar system's ${ }^{56} \mathrm{Fe}$. The requirements that type Ia supernova models make ${ }^{56} \mathrm{Fe}$ and p-nuclei in solar proportions and that they produce $0.5-1.0 M_{\odot}$ of ${ }^{56} \mathrm{Fe}$, and the fact that the typical p-process overproduction factors in this model are $\sim 10^{4}$, lead to the conclusion that the zones that produce p-nuclei comprise $0.04-0.08 M_{\odot}$ of the white dwarf. This is in good agreement with the models (e.g. Khoklov 1990). It appears that type Ia supernovae are capable of producing the bulk of the solar system's p-nuclei.

Another important constraint on the p-process is the presence of live ${ }^{146} \mathrm{Sm}$ in the early solar system (Lugmair et al 1983, Prinzhofer et al 1989). From general galactic abundance evolution arguments, Prinzhofer et al inferred from their measurements that the production ratio of ${ }^{146} \mathrm{Sm} /{ }^{144} \mathrm{Sm}$ should be between 0.07 and 0.5 . This ratio causes problems for the gamma-process for which the production ratio for these isotopes is typically $\sim 0.02$ for the type II model (Wousley \& Howard 1978) and $\sim 0.05$ for the type la model (Howard et al 1991). The inferred production ratio is in fairly good agreement with that found from proton-capture models (e.g. Audouze \& Truran 1975), which led Prinzhofer et al to favor such models for production of p-process elements. As we have seen, however, such models are not astrophysically realistic, so the measurements present a challenge for gamma-process models. Woosley \& Howard (1990) found, however, that if the branching ratios for $(\gamma, n)$ and $(\gamma, \alpha)$ reactions on ${ }^{148} \mathrm{Gd}$ are varied within experimental uncertainties, the ${ }^{146} \mathrm{Sm} /{ }^{144} \mathrm{Sm}$ production ratio could be increased dramatically. It is clearly 
a worthy goal for experimental nuclear physicists to attempt to make accurate measurements of the disintegration rates of ${ }^{148} \mathrm{Gd}$. Another important effect that may alleviate the ${ }^{146} \mathrm{Sm} /{ }^{144} \mathrm{Sm}$ production problem is galactic infall (Clayton et al 1993).

In summary, plausible models exist that do a good job of satisfying most of the constraints on the p-process. The most vexing puzzle that remains is the underproduction of the light p-nuclei. Perhaps, with more work, we will see that type Ia supernovae can produce these nuclei at the correct levels. On the other hand, it may be that we will need to turn our attention to some other site, such as the $\alpha$-process in the mass cut in type II supernovae (Woosley \& Hoffman 1992) or proton-capture reactions in Thorne-Żytkow objects (Cannon et al 1992), to explain the origin of these isotopes.

\section{CONCLUSION}

The terms r-, s-, and p-processes have been successful in clarifying our picture of heavy-element formation. In this paper, we have seen that this is because these different processes reflect the responses a nucleosynthetic system can have to being out of equilibrium. In this way, our understanding of the mechanisms of heavy-element synthesis is firm.

Future work will continue to focus on using all clues at our disposal to resolve the problems surrounding the astrophysical sites for these processes. Important questions we must seek answers to in the near future include: 1. Do nascent neutron star winds have high enough entropies to drive a full r-process?, 2. Do ${ }^{13} \mathrm{C}$ pockets really form in low-mass $A G B$ stars, and do they give the right neutron exposures for the s-process?, and 3. Can type Ia supernovae make the p-nucleus ${ }^{92}$ Mo? That we can ask such specific questions indicates that we have good ideas about the sites for the $r-, s-$, and p-processes. That we do not have answers shows that we have much work to do.

\section{ACKNOWLEDGMENTS}

The author is grateful to G. M. Fuller, W. M. Howard, G. J. Mathews, and S. E. Woosley for discussions and to N. Luo for assistance. He is especially grateful to M. F. El Eid for discussions and for some of the subroutines necessary to compute the results shown in Figures 3,4,5, and 6, and to D. D. Clayton for a critical reading of the manuscript. This work was supported in part by NASA grant NAGW-3480.

Any Annual Review chapter, as well as any article cited in an Annual Review chapter, may be purchased from the Annual Reviews Preprints and Reprints service. 1-800-347-8007; 415-259-5017; email arpr@class.org 


\section{Literature Cited}

Anders E, Grevesse N. 1989. Geochim. Cosmochim. Acta 53:197

Anders E. Zinner E. 1993. Meteoritics 28:490

Arcoragi J-P, Langer N, Arnould M. 1991. Astron. Astrophys. 249:134

Arnett WD, Thielemann F-K. 1985. Ap. J 295:589

Arnould M. 1976. Astron. Astrophys. 46:117

Arnould M. Howard WM. 1993. Meteoritics 28.561

Arnould M, Rayet M, Hashimoto M. 1992. In Unstable Nuclei in Astrophysics, ed. S Kubono, T Kajino, p. 23. Singapore: World Scientific

Audouze J, Truran JW. 1975. Ap. J. 202:204

Baraffe I, El Eid MF. 1994. In Proc. 7th Ringberg Conf. on Nuclear Astrophysics. In press

Baraffe l, El Eid MF, Prantzos N. 1992. Arrmon. Astrophys. 258:357

Baym G. Pethick C. 1975. Annu. Rev. Astron Astrophys. 17:415

Baym G, Bethe HA, Pethick C. 1971. Nucl. Phys. A 175:225

Bazan G, Lattanzio JC. 1993. Ap. J. 409:762

Beer H Macklin RL. 1988. Ap. I. 331:1047

Beer H, Macklin RL. 1989. Ap. J. 339:962

Beer H, Walter G, Macklin RL. 1985. In Capture Camma-Ray Spectroscopy and Related Topics 1984, ed S Raman, p. 778. New York Am. Inst. Phys

Beer H, Walter G, Macklin RL, Patchett PJ. 1984 Astron Astrophys. $211: 245$

Bethe HA. 1990 Rev Mud Phys, 62:801

Bethe HA, Wilson JR. 1985. Ap. J. 295:14

Blake JB, Woosley SE, Weaver TA, Schramm DN 1981. Ap. J. 248:315

Boothroyd AI, Sackmann I-J. 1988a. Ap. J 328:632

Boothroyd Al, Sackmann 1-J, 1988b. Ap. J. 328:641

Boothroyd AI. Sackmann I-J. 1988c. Ap. J 328:653

Boothroyd Al, Sackmann I-J. 1988d. Ap. J. 328:671

Brown LE, Clayton DD. 1992. Ap. J. Lett. $392 \cdot 179$

Burbidge EM, Burbidge GR, Fowler WA, Hoyle F. 1957. Rev. Mod. Phys. 29:547

Busso M, Gallino R. 1985. Astron. Astrophys. 151:205

Busso M, Picchio G, Gallino R, Chieffi A. 1988. Ap. J. $326: 196$

Cameron AGW. 1955. Ap. J. 121:144

Cameron AGW. 1957. Chalk River Rep. CRL41, Atomic Energy Can. Ltd.

Cameron AGW, Cowan JJ, Truran JW. 1985. In Nucleosynthesis: Challenges and New Developments, ed. WD Arnett, JW Truran, 190. Chicago: Univ. Chicago Press

Cameron AGW, Delano MD, Truran JW. 1970 CERN Rep. 70-302:735
Cameron AGW, Thielemann F-K, Cowans JJ. 1993. Phys. Rep. 227:283

Cannon RC, Eggleton PP, Podsiadlowski P, Żytkow AN. 1992. Ap. J. 386:206

Clayton DD. 1978. Moon and Planets 19:109

Clayton DD. 1982. Q.J.R. Astron. Soc. 23:174

Clayton DD. 1988a. MNRAS 234:1

Clayton DD. 1988b. Ap. J. 334:191

Clayton DD. 1989. Ap. J. 340:613

Clayton DD, Fowler WA, Hull TE, Zimmerman BA. 1961. Ann. Phys. 12:331

Clayton DD, Hartmann DH, Leising MD. 1993. Ap. J. Lett. 415:L25

Clayton DD, Rassbach ME, 1967. Ap. J. 148:69 Clayton DD, Ward RA. 1974. Ap. J. 193:397

Colpi M. Shapiro SL, Teukolsky SA. 1989. Ap. J. $339: 318$

Colpi M, Shapiro SL, Teukolsky SA. 1991. Ap. J. $369: 422$

Colpi M, Shapiro SL, Teukolsky SA. 1993. Ap. J. 414:717

Cowan JJ, Cameron AGW, Truran JW. 1980. Ap. J. $241: 1090$

Cowan JJ, Cameron AGW, Truran JW. 1982. Ap. J. $252: 348$

Cowan JJ, Cameron AGW, Truran JW. 1983. Ap. J. $265: 429$

Cowan JJ, Cameron AGW, Truran JW. 1985.Ap. J. $294: 656$

Cowan JJ, Thielemann F-K, Truran JW. 199I Phys. Rep. 208:267

Duncan RC, Shapiro SL, Wasserman I. 1986. Ap. J. 309:141

Eichler D Livio M Piran T, Schramm DN. 1989 Nature 340:126

Epstein Rl, Colgate SA, Haxton WC. 1988. Phys. Rev. Lett. 61:2038

Evans CR, Mathews GJ. I988. In Origin and Distribution of the Elements, ed. GJ Mathews, p. 619. Singapore: World Scientific

Gallino R, Busso M, Picchio G, Raiteri CM, Renzini A. 1988. Ap. J. Lett. 334:L45

Gallino R, Busso M, Raiteri CM. 1990. Nature 348:298

Gallino R, Raiteri CM, Busso M. 1994. Ap. J. Submitted

Gilroy KK, Sneden C, Pilachowski CA, Cowan JJ. 1988. Ap. J. 327:298

Hillebrandt W. 1978. Space Sci. Rev. 21:639

Hillebrandt W, Takahashi K, Kodama T. 1976 Astron. Astrophys. 52:63

Hogan CJ, Applegate JH. 1987. Nature 320:236

Hollowell DE, Iben I Jr. 1988. Ap. J. Lett. 333:L25

Howard WM, Goriely S, Rayet M, Arnould M 1993. Ap. J. 417:713

Howard WM, Mathews GJ, Takahashi K, Ward RA. 1986. Ap. J. 309:633

Howard WM, Meyer BS. 1992. In Nuclei in the Cosmos, ed. F Käppeler, K Wisshak, p. 575 Bristol: Inst. Phys.

Howard WM, Meyer BS, Clayton DD. 1992 Meteoritics 27:404 
Howard WM, Meyer BS, Woosley SE. 1991. Ap J. Lett. 373:L5

Hoyle F, Clayton DD. 1974. Ap. J. 191:705

Iben I Jr. 1975a. Ap. J. 196:525

Iben 1 Jr. 1975b. Ap. J. 196:549

Iben I Jr. 1976. Ap. J. 208:165

Iben I Jr, Renzini A. 1982, Ap J 260:821

Iben I Jr, Renzini A. 1983. Annu. Rev. Astron. Astrophys. 21:271

Ito K. 196I. Prog. Theor. Phys. 26:990

Käppeler F, Beer H, Wisshak K. 1989. Rep Prog. Phys. 52:945

Käppeler F, Gallino R, Busso M, Picchio G, Rai teri CM. 1990. Ap. J. 354:630

Khoklov A. 1990. Astron. Astrophys. 245:114

Klapdor HV, Oda T, Metzinger J, Hillebrandt W, Thielemann F-K. 1981. Z. Phys. A 299:213

Kochanek CS. 1992. Ap. J. 398:234

Kodama T, Takahashi K. 1975. Nucl. Phys. A 239:489

Kratz K-L, Bitouzet J-P, Thielemann F-K, Möller P, Pfeiffer B. 1993. Ap. J. 403:216

Kratz K-L, Harms V, Hillebrandt W, Pfeiffer B. Thielemann F-K, Wöhr A. 1990. Z Phys. A 336:357

Kratz K-L, Thielemann F-K, Hillebrandt W, Möller P, Harms V, Wöhr A, Truran JW. 1988. J. Phys. G 24:S331

Lamb SA, Howard WM, Truran JW, Iben I Jr. 1977. Ap. J. $217: 213$

Lambert DL. 1992. Astron. Astrophys. Rev. $3 \cdot 201$

Lambert DL. 1993. In Origin and Evolution of the Elements, ed. N Prantzos, E VangioniFlam, M Cassé, p. 257. Cambridge: Cambridge Univ. Press

Lambert DL, Gustafsson B, Eriksson K Hinkle KH. 1986. Ap. J. Suppl. 62:373

Langer N, Arcoragi J-P, Arnould M. 1989. Astron. Astrophys. 210:187

Lattimer JM, Mackie F, Ravenhall DG, Schramm DN. 1977. Ap.j. 213.225

Lattimer JM, Pethick CJ, Ravenhall DG, Lamb DQ. 1985. Nucl. Phys. A 432:646

Lattimer JM, Schramm DN. 1974. Ap. J. Lett. 192:L145

Lattimer JM, Schramm DN. 1976. Ap. J $210: 549$

Lattimer JM, Swesty 1991. Nucl. Phys. A $535: 331$

LeBlanc JM, Wilson JR. 1970. Ap. J. 161:541

Lee T, Schramm DN, Wefel JP, Blake JB. 1979 Ap. J. 232:854

Lugmair GW, Shimamura T, Lewis RS, Anders E. 1983. Science 222:1015

Macklin RL. 1970. Ap.J. 162:353

Mathews GJ, Bazan G, Cowan JJ. 1992. Ap. J. $391: 719$

Mathews GJ, Bazan G, Cowan JJ, Schramm DN 1993. Phys. Rep. 227:175

Mathews GJ, Cowan JJ. 1990 . Nature 345.491

McWilliam RA, Lambert DL. 1988. MNRAS 2.30:573
Meier DL, Epstein RI, Arnett WD, Schramm DN. 1976. Ap. J. 204:869

Merrill PW. 1952. Science 115:484

Meyer BS. 1989. Ap. J. 343:254

Meyer BS. 1993. Phys. Rep. 227:257

Meyer BS. 1994. Ap. J. Submitted

Meyer BS, Mathews GJ, Howard WM, Woosley SE, Hoffmann R. 1992. Ap. J. 399:656

Meyer BS, Walsh JH. 1993. In Nuclear Physics in the Universe, ed. MW Guidry, MR Strayer, p. 9. Bristol:Inst. Phys.

Möller P, Nix JR. 1988. At. Data Nucl. Data Tables 39:213

Müller E, Hillebrandt W. 1979. Astron. Astrophys. 88:147

Ott U, Begemann F. 1990a. Ap. J. Lett. 353:L57

OtI U, Begemann F, 1990b Lunar Planet Sci. XXI:920

Prantzos N, Arnould M, Arcoragi J-P. 1987. Ap. J. 315:209

Prantzos N, Hashimoto M, Rayet M, Arnould M. 1990. Astron. Astrophys. 238:455

Prinzhofer A, Papanastassiou DA, Wasserburg GJ. 1989. Ap. J. Lett. 344 L81

Qian Y-Z, Fuller GM, Mathews GJ, Mayle RW, Wilson JR, Woosley SE. 1993. Phys. Rev Lett. 71:1965

Raiteri CM, Busso M, Gallino R, Picchio G. 1991a. Ap. J. 371:665

Raiteri CM, Busso M, Gallino $\mathrm{R}$, Pulone $\mathrm{L}$. 1991b. Ap. J. 367:228

Rayet M, El Eid M, Arnould M. 1992. In Nuclei in the Cosmos, ed. F Käppeler, $\mathrm{K}$ Wisshak, $\mathrm{p}$ 613. Bristol:Inst. Phys.

Raye1 M, Prantzos N, Arnould M 1990 Astron. Astrophys. 227:271

Richter S, Ott U, Begemann F. 1992. Lunar Planet Sci XXIII:1147

Salpeter EE, Shapiro SL. 1981. Ap. J. 251:31

Sato K. 1974. Prog. Theor. Phys. 51:726

Schramm DN. 1973. Ap.J. 185:293

Schwarzchild M, Härm R. 1967. Ap. J. 150:961

Seeger PA, Fowler WA, Clayton DD. 1965. Ap. J. Suppl. 11:121

Smith VV, Lambert DL. 1986. Ap. J. 311:843

Sneden C, Parthasarathy M. 1983. Ap. J. 267:757

Sneden C, Pilachowski CA. 1985. Ap. J. Lett. 288:L55

Srinivasan B, Anders E. 1978. Science 201:51

Symbalisty EMD. 1984. Ap. J. 285:729

Symbalisty EMD, Schramm DN. 1982. Astmphys. Lett. 22:143

Symbalisty EMD, Schramm DN, Wilson JR 1985. Ap. J. Let1. 291:L59

Takahashi K, Witti J, Janka H-Th. 1994. Astron Astrophys. In press

Takahashi K, Yokoi K. 1987. Alom. Data Nucl Data Tables 36:375

Tammann GA. 1982. In Supernovae: A Study of Current Research, ed. M. Rees, R. Stoneham, p. 37I. Dordrecht:Reidel

Tang M, Anders E. 1988. Geochim. Cosmochim 
Acta 52: 1235

Thielemann F-K, Arnould M, Hillebrandt W. 1979. Astron. Astrophys. 74:175

Thielemann F-K, Hashimoto $\mathrm{M}$, Nomoto $\mathrm{K}$. 1990 Ap. J. 349:222

Truran JW, Cameron AGW. 1972. Ap. J. 171:89

Truran JW, Cowan JJ, Cameron AGW. 1978. Ap. J. Lett. 222:L63

Truran JW, Iben I Jr. 1977. Ap. J. 216:797

Tubbs DL, Schramm DN. 1975. Ap. J. 201:467

Ulrich RK. 1973. In Explosive Nucleosynthesis, ed. DN Schramm, WD Arnett, p. 139. Austin: Univ. Texas Press

Van den Bergh S, Tammann GA. 1991. Annu. Rev. Astrunl. Astrophys. 29:363

Walker TP, Steigman G, Schramm DN, Olive KA, Kang H-S. 1991. Ap. J. 376:5I

Wapstra AH, Audi G, Hoekstra R. 1988. At. Data Nucl. Data Tables 39:281

Ward RA, Newman MJ. 1978. Ap. J. 219:195

Ward RA, Newman MJ, Clayton DD. 1976. Ap. J. Suppl. 31:33

Wefel JP, Schramm DN, Blake JB, PridmoreBrown D. 1981. Ap. J. Suppl. 45:565
Weigert A. 1966. Z. Astrophys. 64:395

Wheeler JC, Sneden C, Truran JW. 1989. Annu

Rev. Astron. Astrophys. 27:279

Wilson JR, Mayle RW. 1993. Phys. Rep. 227: 97

Woosley SE, Fowler WA, Holmes JA, Zimmerman BA. 1975. Cal. Inst. Tech., Kellogg Rad. Lab. Preprint No. OAP-422

Woosley SE, Hartmann DH, Hoffman RD, Hax ton WC. 1990. Ap. J. 356:272

Woosley SE, Hoffman RD. 1992. Ap. J. 395:202

Woosley SE, Howard WM. 1978. Ap. J. Suppl. $36: 285$

Woosley SE, Howard WM. 1990. Ap. J. Lett 354:L21

Woosley SE, Mathews GJ, Wilson JR, Hoffman RD, Meyer BS. 1994. Ap. J. In press

Yokoi K, Takahashi K. 1981. In Proc, 4th Int Conf. on Nuclei Far from Stability, CERN Rep. 81-09, p. 351

Zinner E, Tang M, Anders E. 1987. Nature 330:730

Zinner E, Amari S, Lewis RS. 1991. Ap. J. Lett. $382 \cdot \mathrm{L} 47$ 